\title{
Sociocultural Mechanisms of Conflict: Combining Topic and Stochastic Actor-Oriented Models in an Analysis of Afghanistan, 1979-2001 *
}

\author{
Daniel Karell \\ Division of Social Science \\ New York University Abu Dhabi
}

\author{
Michael Freedman \\ Department of Political Science \\ Massachusetts Institute of Technology
}

\section{Pre-print copy}

Published version in Poetics (December 2019)

https://doi.org/10.1016/j.poetic.2019.101403

\footnotetext{
${ }^{*}$ The authors thank Viviana Amati, Ulrik Brandes, Christian Steglich, Brandon Stewart, Blaine Robbins, and the participants of the Workshop on the Frontiers of Network Science (New York University Abu Dhabi, April 2018), including Emily Erikson, James Kitts, and Kinga Makovi. We also thank the anonymous reviewers and the editors for their comments. Finally, we are indebted to Fotini Christia for sharing her data. Any remaining errors and omissions are our responsibility. Research for this project was conducted while the lead author was a Fung Global Fellow at Princeton University's Institute for International and Regional Studies (PIIRS).
} 


\begin{abstract}
How do sociocultural dynamics shape conflict? We develop a relational understanding of how social relations, culture, and conflict are interwoven. Using this framework, we examine how combatants' associations with cultural elements affect the interpersonal relationships underlying conflict dynamics, as well as how these relationships engender associations to cultural elements. To do so, we first introduce a novel analytical approach that synthesizes computational textual analysis and stochastic actor-oriented models of longitudinal networks. We then use our approach to analyze a two-level socio-semantic graph representing both the cultural domain and social relationships of prominent militants operating in one Afghan province, Balkh, between 1979 and 2001. Our results indicate that militants' interpersonal comradeships rely, in part, on their connections to cultural elements and relative power. Comradeship, in turn, fosters militants' connections to cultural elements. We conclude by discussing how conflict studies can continue to build on insights from cultural sociology, as well as how cultural sociology and socio-semantic network research can benefit from further engaging conflict studies and developing our analytical approach. We also highlight provisional insights into endogenous mechanisms of conflict resolution and cultural change.
\end{abstract}

Key words: Socio-semantic networks; topic models; stochastic actor-oriented models; culture; conflict 


\section{Introduction}

How do sociocultural dynamics shape conflict? The contemporary conflict studies scholarship has often overlooked cultural factors, instead focusing on non-cultural drivers of violent contention, such as economic opportunity (Collier and Hoeffler 2004), poverty (Kawalerowicz and Biggs 2015), inequality and exclusion ((Nepal et al. 2011; Cederman et al. 2013; Karell and Schutte 2018) grievances originating in relative or unexpected hardship (Gurr [1970] 2011; Hechter et al. 2016), a weak state (Fearon and Laitin 2003), rebels' strategic reaction to third-party intervention (Crost et al. 2014), and warring parties' pursuit of minimum winning coalitions (Christia 2012).

However, a growing body of research has found that combatants' "distinctive worldviews" (Maynard 2019: 3), or ideologies, ${ }^{1}$ both influence and, in turn, are influenced by conflict (e.g., Gutiérrez Sanín and Wood 2014; Walter 2017a;b; Gade et al. 2019a). Yet, while this scholarship sheds important new light on the link between ideology and violence, it has developed its insights largely within a psychological-behavioral framework. For example, some studies focus on how ideology governs militant groups' behavior, such as their internal cohesion and treatment of civilians (Berman 2009; Walter 2017a). Other work emphasizes that individual militants may adhere to an ideology not only psychologically, because of, for example, sincere commitment, but also simply behaviorally, such as when conforming to social pressure (Maynard 2019). Research using a network approach, thereby situating militants' ideology in a broader ideological field, highlights how ideological proximity influences decisions to enter into alliances or rivalries (Gade et al. 2019a;b).

We develop an alternative understanding of how cultural phenomena, such as ideology, can affect sociopolitical conflicts. To do so, we depart from the current scholarship in two ways. First, we adopt a relational approach. That is, rather than attribute conflict dynamics to characteristics of individuals, we specify the process of interactions - and individuals' interpretations of these interactions - that help drive conflict (Emirbayer 1997; Bearman 2003). Second, we use our relational approach to subsume ideology under culture. Rather than focus on the "worldviews" of individuals, we group these worldviews with other cultural elements - symbols, frames, and discursive topics, for example - that shape the social world through their influence on social relations, which themselves give meaning to the cultural elements (Archer 1998; Mohr 1998; Somers 2008; Pachucki and Breiger 2010; Mische 2011; Breiger and Puetz 2015). ${ }^{2}$ For instance, shared cultural elements, such as musical tastes, can encourage the formation of friendships between people (e.g., Lizardo 2006; Lewis et al. 2012; Lewis and Kaufman 2018), while individuals' social interactions can

\footnotetext{
${ }^{1}$ The ideology and conflict literature conceptualizes ideology broadly (Maynard 2019). Gutiérrez Sanín and Wood (2014: 215), for example, define it as "a more or less systematic set of ideas that includes the identification of a referent group (a class, ethnic, or other social group), an enunciation of the grievances or challenges that the group confronts, the identification of objectives on behalf of that group (political change or defense against its threat), and a (perhaps vaguely defined) program of action."

${ }^{2}$ To paraphrase Fushe and Mützel (2011: 1073), the relational perspective on culture refrains from converting relational properties into individual attributes. Instead, culture can be seen as an "inseparable commingling of network relations and discursive processes ... language and relational ties are fused within a sociocultural setting." This perspective differs from understanding culture as a sticky attribute of groups - an understanding used in arguments that certain cultural values (Benjamin and Simon 2002) and deep-seated, immutable hatreds between cultural groups (Kaplan 1993) cause sociopolitical conflict.
} 
generate links among cultural elements (Fuhse et al. 2019).

A focus on culture's relational dynamics affords new opportunities for understanding how the interdependence between a range of ideations and social relations affects conflict. However, its embrace of sociocultural complexity has the potential to obfuscate rather than clarify. Fortunately, cultural sociology and sociosemantic network research have been developing a theoretical and analytical approach for fruitfully studying this complexity by conceptualizing the entire system as a network graph. Such conceptualizations enable the use of network analysis methodologies to identify the dynamics properties of sociocultural space (e.g., Mohr and Duquenne 1997; Roth and Cointet 2010; Roth 2013; Nerghes et al. 2015). For example, exponential random graph models (ERGMs) can be used to test hypotheses of how sociocultural space may take shape while controlling for alternative arguments (e.g., Basov and Brennecke 2017; Basov 2018). Similarly, stochastic actor-oriented models (SAOMs) help analyze concurrent network mechanisms over time to isolate the causal direction of sociocultural processes (e.g., Lewis et al. 2012; Edelmann and Vaisey 2014; Lewis and Kaufman 2018).

We adopt the socio-semantic network approach to examine how combatants' interpersonal relations interweave with connections to culture, and how this interweaving shapes conflict. Specifically, we develop answers to two questions. First, how do combatants' connections with discursive topics affect their formation of comrade relations with other combatants? Second, because combatants are known to adopt different rhetoric throughout a conflict (Christia 2012; Isaacs 2016), we ask: How do comrade ties among individual combatants affect their connections to discursive topics? We pay special attention to the formation of comradeship, or affinity, among combatants because of its potential to promote conflict resolution. Greater feelings of commonality decrease tolerance of collateral casualties (Schori-Eyal et al. 2019), while unity among combatants helps shorten civil wars and insurgencies (Cunningham 2006; Cederman and Vogt 2017; Gade et al. 2019a) and diminish the likelihood of conflict recurrence (Blattman and Miguel 2010). Moreover, by focusing on the sociocultural dynamics that generate relations of affinity rather than on interpreting cultural content, our study helps advance the formal analysis of culture by specifying potentially general mechanisms (Martin 2002; Edelmann and Mohr 2018). ${ }^{3}$

Our socio-semantic network analysis examines the sociocultural links among militants in one war-torn Afghan province, Balkh (1979 to 2001), as a multilevel graph. A multilevel graph helps us depict three distinct but interlinked networks: social actors and their relationships; cultural elements and how they are bound together; and the ties between actors and cultural elements (Basov et al. 2017; Basov 2019). The analysis is guided by a novel explanatory argument drawing on the conflict studies, relational sociology, and the social movement literatures. Namely, we build on Mische and White's (1998) insight that interpersonal encounters can lead to narratives of affinity - in our case, comradeship between Blakh's militants - but not necessarily so (see also Karell and Freedman 2019). At times, these encounters can become "situations", during which contention emerges between individuals. We argue that situations are less likely, and, thus,

\footnotetext{
${ }^{3}$ Following Hedström and Ylikoski (2010), we conceive of mechanisms as specific interactions between entities that bring about social change.
} 
interpersonal affinity more likely, when individuals share cultural connections and enjoy symmetrical power. Our results offer evidence in support of this argument. They additionally show how social relationships of affinity help engender individuals' connections to cultural elements.

The finding that shared associations with cultural elements can, under certain conditions, lead to comradeship among militants, which, in turn, promotes ties to cultural elements, points towards a key insight into conflict resolution. By initially encouraging shared cultural connections, it may be possible to spark an endogenous process that generates increasing unity among combatants during times of conflict. We elaborate this insight in our Discussion section. We also use the Discussion section to explore what our results suggest about endogenous cultural change (Kaufman 2004).

This article helps expand both the conflict studies and socio-semantic network scholarship. It offers the conflict studies literature evidence of several relational micro-level mechanisms driving conflict dynamics over time, as well as a framework for broadening its theoretical and empirical analysis of culture's role in conflict. While this framework differs from recent conflict studies research, it advances a sociological tradition of understanding sociopolitical violence through the interaction of individuals' social networks and the cultural meanings they carry into settings of contention (e.g., Bearman 1993; Gould 1995; 1996).

At the same time, the article's examination of sociocultural mechanisms during conflict facilitates an analysis of how relative power shapes socio-semantic dynamics, particularly the conversion of shared discursive topics into ties of affinity. In addition, the study introduces a novel analytical strategy for using data from large bodies of text in conjunction with SAOMs to examine the effect of sociocultural mechanisms over time. This "text-SAOM" design is also applicable to a range of socio-semantic network research areas, such as the joint evolution of social and cultural space (Fuhse et al. 2019) and mechanisms driving endogenous cultural change (Kaufman 2004).

\section{A relational view of culture and conflict}

Gould (2003) offered one of the clearest articulations of the relational view of conflict in his study on contention: people and small groups tend to engage in violence when ambiguity exists over perceived relative social rank. That is, conflict is a function of symmetrical status or power relations between actors. Violence, in Gould's empirical cases, is used to establish a hierarchy Christia (2012) developed a similar argument in her study of militant groups operating during the Afghan conflict, observing that groups' alliances were a function of power hierarchies. During an ongoing realignment of coalitions, increasingly powerful groups sought alliances with weaker ones, and vice versa.

Building on the foundational insight that individuals' positions vis-à-vis others can induce contention, we expect that the similarity between a militant's connections with cultural elements and those of another militant will affect whether they develop interpersonal relationships of affinity or animosity. At the same time, we expect that these social relations of affinity and animosity influence militants' associations with the 
cultural space. We elaborate on these expectations by describing specific mechanisms underlying these two processes.

First, with regards to the formation of interpersonal relationships, individuals - in our case, militantswill be drawn into encounters with one another based on shared connections and familiarity with specific cultural elements. During these encounters, they can use their shared knowledge of cultural elements to engage in appreciation and discussion (Vaisey and Lizardo 2010; Lewis et al. 2012; Edelmann and Vaisey 2014), eventually crafting a narrative of affinity for one another and forging a tie of comradeship (White 2008). Affinity typically emerges in these circumstances because individuals tend to foreground their shared cultural familiarity while suppressing distinct perspectives that might generate tension during the encounter (White 1995; Mische and White 1998; Mische 2007; 2011). For example, two militants might engage in discussions over how the institutions of international politics - one of the discursive topics we uncoverfacilitate imperialistic behavior by Western countries while sidestepping potential disagreement over whether both the United States and Soviet Union or just the Soviet Union are imperialist.

However, the formation of friendships from shared cultural connections is not a given. Individuals could opt not to deaden differing views or misgivings. Instead, the encounter could be used as an opportunity to articulate objections rooted in aspects of the individuals' self-narratives that clash. As a result, a perception of interpersonal animosity could emerge (Mische and White 1998; White 2008; Karell and Freedman 2019). Such instances of disruption - what Mische and White (1998) call "situations" — are likely to occur, we argue, when power is asymmetrical.

Under conditions of asymmetrical power, powerful individuals would likely find it easier to assert contradictory viewpoints or critique their interlocutors' worldviews and self-narratives, thereby spurring objections by the interlocutor. Returning to our example, a relatively powerful militant would feel more confident in declaring that $\mathrm{e}^{4}$ has unique insight into the inherent imperialistic tendencies of capitalism and claim that both the United States and Soviet Union are pursuing imperial goals, despite his interlocutor believing that only the Soviet Union is imperialistic due to its invasion of Afghanistan in 1979. Such a disagreement would make comradeship less likely.

Conditions of equal power, in contrast, increase the likelihood that any potential critiques would be held "in abeyance" (Mische and White 1998: 711). Individuals would find it less easy to assert distinct views over an equally powerful alter. This reasoning - effectively an interaction of shared cultural connections and relative power-helps explain why similarly powerful and influential militants operated as part of the same organization in Afghanistan, despite Gould's (2003) argument that symmetrical power leads to contention. Shared cultural associations would be drawing them together while similar levels of power suppress potential disagreements. If the militants stopped sharing cultural connections, we would expect, like Gould, to see conflict.

Our reasoning also leads us to our first hypothesis:

\footnotetext{
${ }^{4}$ When referring to militants, we use the masculine pronoun here and throughout the article because all individuals in our data are men.
} 
H1. The more militants are linked to the same cultural elements, the more likely they are to form comrade ties, as long as they hold the same level of power.

Our reasoning additionally implies that militants linked to the same cultural elements, but with differing levels of power, are less likely to be comrades.

How do individuals come to be connection to cultural elements? Militants are known to change the rhetoric and ideology they espouse during the course of a conflict (Christia 2012; Isaacs 2016). Cultural sociology suggests that such change depends, in part, on shifts in militants' interpersonal relations (Fuhse et al. 2019). Building on this insight, we posit that an expanding set of friendly relations encourages individuals to explore, adopt, and become associated with unfamiliar pieces of the broader culture. Friendly relations help individuals "conceive of the possibility of alternative [cultural elements than what exists in] the status quo" (Martin 2002: 874), which promotes new connections with elements of the cultural space. For example, as a militant primarily interested in international politics gains more comrades, he is more likely to become familiar with new aspects of the surrounding culture, such as religious codes (another cultural element we uncover). Thus, we hypothesize:

H2. The more militants receive comrade ties, the more likely they are to establish new connections to cultural elements.

To reprise, we draw on insights from conflict studies and relational and cultural sociology to posit two sociocultural mechanisms driving socio-semantic conflict dynamics. Namely, the more two militants share connections to cultural elements, they more likely they will become comrades - but only when they also have similar levels of power. The establishment of this comrade tie then exposes the militants to new cultural elements, increasing the likelihood they will become familiar with more elements of the broader culture. Of course, other mechanisms may concurrently encourage militants to form ties of affinity - and animosity - as well as connections to cultural elements. Here, we focus on the interplay of friendly relations and discursive topics. We view this as an early step in helping advance a socio-semantic network research agenda on conflict dynamics. We anticipate that further research will uncover other mechanisms that together can offer a more comprehensive understanding of culture and conflict.

\section{Analytical strategy}

Analyzing sociocultural conflict dynamics requires an analytical approach that, to paraphrase Lewis and Kaufman (2018: 1686), can: specify cultural space without presuming labels for its elements or salient dimensions of categorization; identify and disentangle multiple mechanisms generating (or dissolving) connections among cultural elements and social actors, including mechanisms that interact with actors' attributes; and capture the interdependencies between cultural and social domains (see also Brandes et al. 2013; Wang et al. 2013). Moreover, the approach must jointly model each of these components longitudinally to examine 
how sociocultural relations are "continuously created, reproduced, and modified" (Fushe and Mützel 2011: 1078), as well as to clarify the direction of causation.

Achieving each of these aspects in one analytical approach is not as simple as it might seem. The specification of cultural elements, for example, often relies on close-ended survey instruments or assumed categories that impose interpretations on the data that may not be relevant (Erickson 1996; Lewis and Kaufman 2018). A body of work on networks and culture provides a useful alternative approach: researchers draw on case-specific expertise to code cultural elements - often, words in a text - and their relationships, thereby rendering systems of meaning as network data (e.g., Mohr 1998; Bearman and Stovel 2000; McLean 2007). But this approach raises new questions. What are good strategies for validating the coding of the cultural network and for enabling falsifiability and reproducibility? How scalable is expert manual coding to large textual databases, which are of growing interest to social scientists (Nelson 2017)?

Moreover, while previous socio-semantic network research has produced inspired ways to model and evaluate the mechanisms engendering sociocultural ties, our criterion of an analysis of multiple mechanisms operating within and between cultural and social domains is achieved relatively rarely. For example, existing studies examine a few mechanisms independently, rather than with respect to one another (e.g., Roth and Cointet 2010; Roth 2013). Others convert semantic elements into attributes of actors, thereby limiting mechanisms to one level of what could alternatively be conceptualized as a multilevel graph (e.g., Crandall et al. 2008; Lewis et al. 2012; Edelmann and Vaisey 2014). A recent innovation by Basov and Brennecke (2017) models simultaneous mechanisms while respecting the dual nature of sociocultural space - shared meanings are reflected in interpersonal ties - but it does not capture multilevel structure.

While other recent work has made advances in the study of network mechanisms between different domains, there remains room for development. For instance, Hollway et al. (2017) apply a longitudinal view to multilevel graphs, but they focus on organizational ties rather than semantic space. Lomi and Stadtfeld (2014) link individuals to cultural elements - musical tastes - but measure these elements with categories on surveys (returning us to an earlier critique). Basov (2019) inductively identifies semantic elements in a socio-semantic multilevel graph, but the mechanisms of interdependency between domains, or levels, are not analyzed longitudinally.

Indeed, time presents a final challenge to achieving our necessary criteria. Previous temporal approaches in the socio-semantic network literature make strong assumptions about the changing composition of populations under study, limiting their applicability (e.g., Roth and Cointet 2010; Roth 2013), or rely solely upon observations of discrete time slices (e.g., Nerghes et al. 2015). The temporal variants of models commonly used to study network mechanisms, like temporal exponential random graph models (TERGMs), also rely upon cross-sectional observations without modeling the intervening time. As a result, their estimates are sensitive to the length of time between observed periods (Block et al. 2018). 


\subsection{Topic models and stochastic actor-oriented models}

In light of these challenges, we develop a novel analytical approach that synthesizes computational textual analysis, specifically structural topic models (STMs), and stochastic actor-oriented models (SAOMs) of longitudinal networks. Structural topic models, along with other unsupervised topic models of text, provide an opportunity to inductively or abductively define the semantic level with minimal input from researchers' biases and natural inconsistency in coding (Grimmer and Stewart 2013; Nelson 2017; Karell and Freedman 2019). They use the co-occurrence of words appearing in each document within a corpus to identify "topics" that are conceived as having formed the documents. That is, topics are collections of words, varying in frequency, that "hang together" across documents. As a result, topics typically differ from one another in terms of their content and prevalence both within documents and across the corpus (Blei et al. 2003; Grimmer and Stewart 2013).

Topics, as pointed out by Mohr and Bogdanov (2013), can be interpreted as "categories of meaning", or latent ideas forming a conceptual level higher than that of individual words. For socio-semantic network scholars, uncovering topics in textual data offers an opportunity to understand a cultural space of interest by jointly interpreting four results from the topic model: the words within a topic; the frequency of each word within a topic; the prevalence of topics; and the associations between these topics.

Topic models also provide opportunities for validation, falsification, and replication. For example, the polysemy of each word can be measured and compared against theoretical expectations. ${ }^{5}$ Researchers can also check whether topics vary over time in expected ways and whether the results are robust to using different numbers of topics (Lucas et al. 2015; Wilkerson and Casas 2017). If researchers begin the project with a theory in mind, they can posit topical content and prevalence before estimating the topic model, then assess whether the output of various model specifications - variations based on the number of topics to be estimated, temporal dependencies (Blei and Lafferty 2006), and the incorporation of document metadata (Roberts et al. 2014), for example - aligns with the theoretical expectations. Alternatively, researchers could also split their textual data into training and test samples, then use the training data to build and revise the operationalization of culture, while the test sample could be used for falsification (Egami et al. 2018). Each of these decisions would be written into the model specification and programming code, making the process fully reproducible (Nelson 2017).

In our analysis, we define a discursive, or cultural, domain using topics and the relationships among topics. We then link the discursive domain to the social domain - militants and their interpersonal relations - using the authorship of documents. We explain these steps in more detail in the Data and Network Construction section below. The result is a multilevel graph (Wang et al. 2013) that, in our case, is also multiplex because it includes militants' ties of comradeship and enmity (see Huitsing et al. 2014).

Next, we use SAOMs to examine multiple mechanisms driving changes in the multilevel socio-semantic

\footnotetext{
${ }^{5}$ One option to measure words' polysemy is the simplified frequency-exclusivity (FREX) score, which provides the harmonic mean of a word's probability of appearing in a topic and its exclusivity to that topic (Roberts et al. 2014).
} 
graph over time. These models identify the regularities, or mechanisms, that shape networks over time by comparing actors' formation or maintenance of ties to the dissolution or absence of tie formation (Steglich et al. 2010). This tie formation (or no formation) is modeled as conditional on actors' context of existing relations (Lewis and Kaufman 2018; for a theoretical discussion, see Emirbayer 1997; for mathematical details, see Ripley et al. 2019 and Appendix A), which is particularly useful in the case of multilevel graphs because we can estimate which regularities occur in one level, dependent on actors' ties to another level. For example, Hollway et al. (2017) examine how hospitals' decisions to form collaborative relations with other hospitals rely not only on existing collaborations, but also on their links to specific medical specialties (see also Snijders et al. 2013; Lomi and Stadtfeld 2014).

Another advantage of SAOMs is their treatment of time as continuous. This enables the identification of causal direction while also reducing the results' sensitivity to the duration of time between observations (Block et al. 2018). Moreover, modeling continuous time aligns with formative theories of sociocultural space - theories that conceptualize the mutual constitution of social and cultural domains as a continuous process during which both actors and cultural elements are realized and reshaped (Emirbayer 1997; McLean 2007; White 2008; Pachucki and Breiger 2010; Mische 2011).

Stochastic actor-oriented models capture continuous time by simulating network change during the "periods" that occur between observed states of the graph, or "waves". The simulations are sequences of micro-changes to the graph during which there occurs the discrete addition or dissolution of a tie, or the maintenance of an existing tie (or no-tie), between one node and another. ${ }^{6}$ The simulated trajectory of the graph is fit as closely as possible to the observed data, and the estimated parameter values indicate the probability that modeled mechanisms shape the graph's evolution (Snijders 2001; Snijders et al. 2010; Ripley et al. 2019). As Lewis and Kaufman (2018: 1698) describe it, SAOMs identify "the 'movie' (parameter values) that best matches our array of 'still frames' (panel waves)."

Alongside the advantages afforded by our analytical approach are two possible critiques. First, SAOMs may not, at first glance, appear applicable to the semantic domain. Stochastic actor-oriented models are widely understood as assuming that nodes have agency in making decisions, and discursive elements obviously do not have agency. However, we argue that the "actor-oriented" nature of SAOMs in fact make them well suited for analyzing semantic structure. In brief, SAOMs capture thick dependencies (Block et al. 2016) in the semantic level of our grpah in a way that approximates theoretical conceptualizations of the discursive domain as a space saturated with embeddedness. We elaborate on this reasoning in Appendix A.

A second critique is that the results of SAOMs are challenging to interpret. Many effects of interest in SAOMs are interactions, which, because SAOMs take the form of logistic regression, can influence the probability of the outcome differently across values of the data. Moreover, multilevel SAOMs (as well as multilevel ERGMs) rely on interaction terms to capture many of the interdependencies across levels (Wang

\footnotetext{
${ }_{6}^{6}$ The micro-changes rely upon two functions, one for selecting a node to initiate the change (or no change), and a second that estimates what type of change is likely.
} 
et al. 2013; Ripley et al. 2019). ${ }^{7}$ As a result, the coefficients of interactions estimated by our models "do not necessarily provide accurate information about the significance, magnitude, or even the direction of the underlying interaction effect on the predictions" (Mize 2019: 112; see also Ai and Norton 2003; Mood 2010). ${ }^{8}$

To facilitate a meaningful interpretation of our findings, we first present our results as log-odds, which is the common practice. Then, using a range of observed and plausible values, we show how the focal interaction affects the probability of creating or maintain ties, or what is known as the "objective function" of a SAOM (Ripley et al. 2019: Section 13.3). We determine the contribution to the objective function by calculating the linear combination of the interaction effect and its constitutive terms, an increasingly common technique for demonstrating how the effects of model terms can differ across the data space (e.g., Leszczensky and Pink 2019; Snijders and Lomi 2019).

Our approach is not the only way to study socio-semantic network dynamics over time. Rather, we see it as a starting point for gaining two key analytical advantages. First, it helps us inductively or abductively define semantic space from a large body of textual data while allowing for assessments of validity, falsifiability, and reproducibility. Second, it enables us to uncover mechanisms explaining, first, how actors and semantic elements have interwoven trajectories through a sociocultural space, and, second, how they define and change this space as they move through it together (Hollway et al. 2017: 188, 192). Studying socio-semantic graphs in this way helps align empirical analyses with theories of interdependent social and cultural dynamics.

\subsection{The Afghan conflict in Balkh}

We use our analytical approach to examine a two-level graph representing social and semantic relationships among all prominent militants in one Afghan province, Balkh, between 1979 and 2001. This span of time covers four stages that are common in protracted conflicts (Christia 2012): the rise of armed resistance against a regime; the maturation of insurgent groups, possibly with the help of foreign backers; complex, violent contention between these groups; and the emergence of a grinding war between a few forces that have managed to become dominant.

In the case of Afghanistan, the first two stages are widely known as the early and late Jihad. The former is marked by the invasion of the Soviet military to bolster a communist regime under threat from protesters and the early formation of resistance groups. The latter begins when the United States and its allies start supplying the resistance groups with weapons and other resources, enabling them to mount an organized insurgency against the state and Soviet forces. Following the withdrawal of the Soviets in 1989, the conflict evolved into a civil war, during which the communist government collapsed and the militant groups began

\footnotetext{
${ }^{7}$ Note that statistical packages used to estimate one-level SAOMs and ERGMs, such as RSiena (Ripley et al. 2017), often have native effects for single-level networks that are also interaction terms.

${ }^{8}$ Indeed, the editors of the American Sociological Review recently recommended that researchers refrain from "us[ing] the coefficient on the interaction term to draw conclusions about significance of statistical interaction in categorical models" (Mustillo et al. 2018: 1282). Furthermore, the differing effects across the data space means that odds ratios do not solve the problem of interpreting logistic coefficients (Mize 2019). See Indlekofer and Brandes 2013 for a discussion on using odds ratios to interpret $\mathrm{SAOM}$ results.
} 
Table 1: Characteristics of Militants in Balkh

\begin{tabular}{ll}
\hline Number of individual militants & 55 \\
Unique group affiliations & 12 \\
Unique ethnicities & 6 \\
Militants who are Sunni Muslim & $48(87 \%)$ \\
Median number of soldiers under militants' command (95\% interval) & $75(0,4250)$ \\
\hline
\end{tabular}

Notes: Identified ethnic groups: Arab; Hazara; Pashtun; Tajik; Turkmen; and Uzbek. All militants are Muslims; if not of the Sunni sect, they are of the Shia sect.

fighting one another for control of the country . Finally, a group of insurgents and religious figures form the Taliban movement in the south of the country and gradually move north, wresting control from the remaining militant groups (Goodson 2001; Zaeef 2010). Their advance is halted by the United States and NATO after the terrorist attacks on September 11, 2001. ${ }^{9}$

Balkh, sitting along Afghanistan's northern border, experienced each of these stages. Local militant commanders rose to prominence in the area during the early and late Jihad, and their fighters engaged in battles with other militant groups throughout the civil war (Rubin 2002). During the Taliban era, alliance formation, betrayals, and battles preceded the fall of the regional urban center, Mazar-i-Sharif, to the Taliban in 1998 (Rashid 2000). Our data of Balkh's militant network captures 55 individuals, belonging to 12 different groups, making 37 changes in comradeship relations and 13 changes in enemy relations across the four stages. We observe 156 changes in militants' connections to discursive topics, reflects the commonly observed pattern of combatants espousing a variety of ideas (Christia 2012; Isaacs 2016), such as the details of battles, revolutionary visions of the future, and the doctrines of fundamentalist Islam (Karell and Freedman 2019). Table 1 summarizes our data on militants.

While Balkh experienced the general rhythm of protracted conflict, it was not representative of the entire country. For example, it did not have the tribal structure that shaped the battles in the south (Martin 2014). Yet, Balkh was a setting in which typical general conflict dynamics unfolded: fighters belonged to ethnic and militant groups; they joined and switched alliances; they had varied levels of resources; and their membership in local units of large militant groups exposed them to the general discourse pervading the conflict (Rubin 2002; Zaeef 2010; Christia 2012). Consequently, instead of conceiving of Balkh as representative of the entire Afghan conflict, we see the province as a typical case of irregular violent sociopolitical conflict, such as insurgencies and civil wars. As such, it is best suited for the testing of hypotheses (Gerring 2007).

\subsection{Data and network construction}

We observe our multilevel graph during each of the four stages, or waves, common during irregular sociopolitical conflict. The graph's social level comprises directed ties of interpersonal affinity and animosity"comrade" and "enemy" relations - between militants. The two kinds of ties are coded using information

\footnotetext{
${ }^{9}$ We mark these periods with the follow years: early Jihad is 1979 to 1985 ; late Jihad is 1986 to 1989 ; civil war is 1990 to 1993 ;
} Taliban period is 1994 to 2001 . 
gathered during Christia's (2012) interviews with notable militants and political leaders in Balkh. If the interview data indicated that a militant was an ally, friend, superior (in a combat unit), or client (in a patron-client dyad) of another militant, we inferred a comrade tie from the militant to the alter. ${ }^{10}$ If a militant was an enemy of another, we marked an enemy tie. There are 55 actors - the full set of prominent militants operating in Balkh - with seven eventually dropping from the dataset by the final wave, typically because they are killed (Table 2). The interviews also provided information on individuals' ethnic affiliation and their membership in a militant group. In addition, the number of foot soldiers under each militant's command, shown in Table 1, was coded using historical sources (Christia 2012). For more details on the interview data, see Appendix B.

The cultural — or, in our case, semantic or discursive - level is defined by 10 discursive topics in a corpus of publications printed by Afghan militant groups between 1979 and 2001. The corpus contains over 23,000 pages from magazines, pamphlets, speeches, and media reports. Karell and Freedman (2019) identified the topics using a computational abductive analysis. To do so, they first estimated an STM with a 10 topic solution over the entire corpus. They selected this solution based on a qualitative assessment of the results and the joint maximization of exclusivity and semantic coherence (Grimmer and Stewart 2013; Roberts et al. 2014). Then, they inferred and interpreted the topics in light of previous arguments in the relevant literature. When some topics did not fit with these arguments, they engaged an expanding body of scholarship, moving between their results, the literature, and theorization until the topics were individually and jointly conceptualized.

The STM used to identify the topics included temporal, language, and publishing group covariates. As a result, the model uncovers the topics that anchored the insurgents' general semantic field throughout the two decades of conflict while allowing individual topics' prevalence to vary across stages of the conflict. Since our aim is to examine the structure and mechanisms of cultural dynamics rather than the substance of cultural content (see Martin 2002; Edelmann and Mohr 2018), we direct readers interested in the topical content to Appendix C and Karell and Freedman 2019.

The 10 topics are the nodes in our discursive level. To specify relationships between these nodes, we first recover the estimated prevalence of each topic in each document - in our case, pages - per temporal stage (see Farrell 2016; Light and Odden 2017). Then, we calculate the correlation between pairs of topics across all documents in a given stage. So, for example, the more two topics appear together across documents published during the civil war, the larger a positive correlation value between those topics at that time (see Appendix D for details). Following Light and Odden (2017), we code an undirected edge between two topics if the mean correlation is above $0.01 .{ }^{11}$ By repeating this procedure for each stage of the conflict, we capture

\footnotetext{
${ }^{10}$ We remain agnostic about the differences between ally, friend, combat superior, and client, in part because the different connotations in English do not perfectly map onto the connotations in Dari and Pashto. We suggest that future research disaggregate our comrade tie and analyze how each constitutive relation may (or may not) be influenced by distinct mechanisms.

${ }^{11}$ We select 0.01 as the correlation threshold for two reasons. First, we are primarily interested in positive correlations. Theorizing strong non-relations among topics, or why topics are unlikely to appear together across documents, is beyond the scope of this article. Second, we expect that values between zero and 0.01 would reflect noise rather than a true signal,
} 
observations of the semantic network over time (Table 2).

The final network in our graph links militants and topics. To specify these ties, we first identify militants' insurgent group memberships and each group's documents. Then, we connect individual militants to their group's set of documents per stage. In other words, a militant becomes linked to a set of documents through his group membership and his group's authorship of documents. Next, for each militant, we calculate the mean prevalence of each of the 10 topics in his set of documents. If a topic has a prevalence of over 10 percent, we designate a tie from the militant to that topic. We select a threshold of 10 percent because it indicates a prevalence greater than what the prevalence would be if the 10 topics were equally distributed across the text. The edges are directed from militants to topics, indicating that individuals are forming affiliations to cultural elements, and not the other way around. ${ }^{12}$ We repeat this procedure for each stage, thereby observing a militant-to-topic network over time (Table 2).

Our decision to link individuals to discursive topics through their group memberships rests on the fact that in Afghanistan, between 1979 and 2001, much of the conflict-related discourse was developed and spread by national-level militant groups. Militants at the local level would perceive each other as affiliated with specific discursive topics based on their membership in a local level unit of a national group. However, militant groups and discursive topics did not overlap perfectly. A militant could belong to one group but be connected to many topics. Similarly, militants belonging to different groups could be connected to the same topic. For example, members of two rival groups, Jam'iyyat-i Islami and Hizb-i Islami (Gulbuddin faction) were both associated with a topic capturing notions of religiously framed armed resistance, or "jihad" (Rubin 2002; Karell and Freedman 2019).

Figure 1a illustrates how social, organizational, and cultural structure are connected, but not deterministic. Indeed, we see evidence of distinct organizational and cultural structure if we plot militants, groups, and topics as different levels of a multilevel graph. Figure $1 \mathrm{~b}$ shows that groups and topics are not structurally equivalent, indicating that militants' connections to the cultural domain of conflict do not perfectly align with their group membership. ${ }^{13}$

In sum, our two-level socio-semantic graph is made up of four networks: two networks among individual militants, one representing relationships of affinity and the other capturing relationships of animosity; a network linking militants to discursive topics; and a semantic network of discursive topics and their associations. We observe these networks during each stage of the conflict. Summary statistics of the networks are presented in Table 2. Table 3 summarizes network changes in between stages. The networks are graphically

whereas values above 0.01 show us the "backbone" of the discursive structure (Light and Odden 2017: 14). In our case, the mean value of positive correlations is 0.45 ; the standard deviation is 0.43 . Using the threshold of 0.01 removes seven percent of our observations - the weakest positive correlations. In future studies adopting our approach, we expect that researchers' theories will help determine which correlation values should be used to encode edges. For example, theories about weak and strong ties have clear implications for thresholds, and research questions about concepts being unlikely to be discussed together could make use of negative correlation values and approaches for examining signed ties.

${ }^{12}$ Future studies might have theoretical reasons for interpreting ties as directed from topic to actor, or as undirected. Since our study is the initial application of our analytical approach, we make the simple assumption that people decide to become associated or familiar with topics.

${ }^{13}$ In models not presented here, we repeat our main analysis with a multilevel graph that includes groups as their own level, in addition to the social and semantic levels, as depicted in Figure 1b. The core findings remain unchanged. 
Figure 1: Individuals' Links to Groups and Discursive Topics

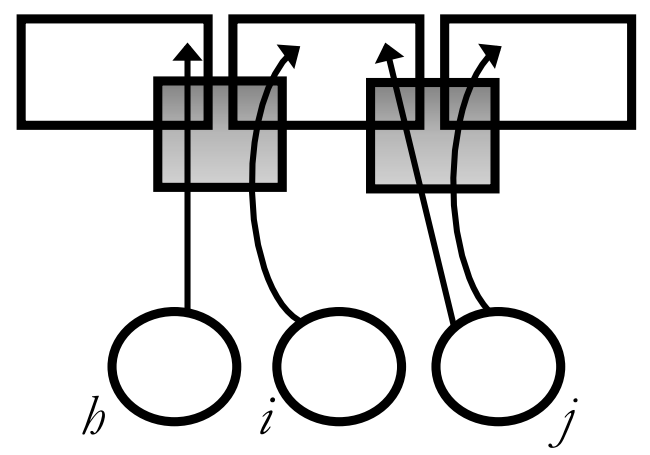

(a) Schematic of how individuals can be linked to discursive topics. Circles represent individuals, squares represent groups, and rectangles represent topics. Note how militants $h$ and $i$ are linked to distinct topics through their shared group membership, and how $i$ and $j$ share a topic but are members of different groups. Also note how militant $j$ is linked to multiple topics through one group.

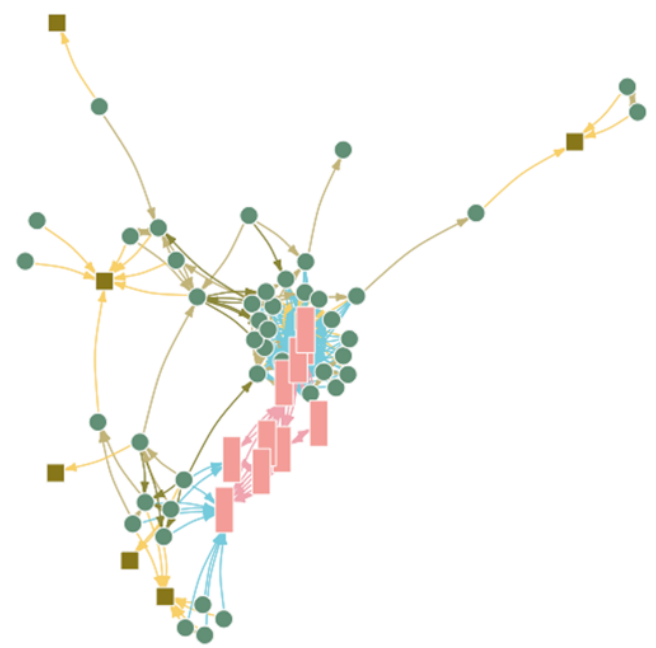

(b) Militants, militant groups, and topics during the early Jihad (Stage 1). Circular vertices represent militants, squares represent groups, and rectangles represent topics. Edges are directed. Note that the groups and topics are not structurally equivalent.

depicted in Appendix E. Appendix D reviews key researcher decisions made during data collection and network construction.

A potential limitation of our data is that the social network is based on militants' recollections. Asking individuals to recall social relations may produce inaccurate depictions of specific interactions (Bernard et al. 1979/1980; Killworth and Bernard 1979/1980). But, recall has been found to accurately and robustly capture patterns of interpersonal relations (Romney and Faust 1982; Freeman and Romney 1987; Freeman et al. 1987), alliances and divisions (Freeman et al. 1988), and strong binary ties (Ferligoj and Hlebec 1999; Hlebec and Ferligoj 2001). This latter research increases our confidence that militants' recall of friends and enemies across well-defined phases of the conflict, rather than during specific events, produces accurate networks.

The discursive network faces a different limitation: historical sources can introduce bias through systematic survival. In our case, Karell and Freedman (2019) addressed this source of bias by collecting publications by nearly all known militant organizations, including near complete runs of publications by the most influential groups. Moreover, this limitation comes with an advantage. Inferring discursive topics from historical data helps mitigate concerns over common response errors, such as social desirability bias. 


\subsection{Model specification}

Our SAOMs contain several "effects", akin to independent variables and covariates, capturing our hypothesized mechanisms and interdependencies across networks in the socio-semantic system. These effects estimate the probability of individuals forming or maintaining a tie, relative to dissolving a tie or maintaining the absence of a tie, in a given "dependent network". A multilevel graph, like ours, may have multiple dependent networks. In this section, we first identify these dependent networks, then explain our independent and key covariate effects. Overall, we include these effects in our models because they test a hypothesis or follow from the general methodological advice on SAOMs (Snijders 2001; 2005; Snijders et al. 2010; Ripley et al. 2019). We end this section by outlining our modeling sequence.

\subsubsection{Dependent networks}

The dependent variables of primary interest are ties in the comrade social network (i.e., affinity between individual militants) and the militant-to-discursive topic network (i.e., links between individual militants and topics). Ties in the enemy social network and discursive network also function as dependent variables because we include both militants' interpersonal ties of enmity and relations among discursive topics to obtain a more valid model. These latter two networks help us account for the effect of, say, a militant's existing enemy ties on the future formation of comrade ties, as well as how cultural elements' interconnections influence individuals' connections to these elements. The models estimate the probability of comrade social ties, enemy social ties, militant-to-topic ties, and inter-topic ties being created or maintained, relative to terminating a tie or maintaining the absence of a tie.

\subsubsection{Independent effects}

We include two explanatory effects that correspond to each of our hypothesized mechanisms. The first effect captures the tendency to form comrade ties based on agreement among militants in terms of their ties to discursive topics (i.e., structural equivalence with respect to their links to cultural elements), conditional on militants sharing similar levels of power (H1). Following Christia (2012) and Gade et al. (2019b), we measure power using the number of soldiers under a militant's command. ${ }^{14}$ The second effect, corresponding to our second hypothesis (H2), models the relationship between the number of comrade ties a militant receives and his subsequent formation of links to discursive topics. Table 4 presents graphical depictions of the effects.

\footnotetext{
${ }^{14}$ There are other sources of power, such as financial resources and territorial control. However, in the Afghan case, the number of soldiers under command is a good indicator of individuals' power. Money was necessary to recruit and retain soldiers, making the number of soldiers a proxy of finances and other resources, as Gade et al. (2019b) point out, whereas multiple individual commanders often shared operational areas.
} 
Table 2: Summary Statistics of Networks in Multilevel Graph

\begin{tabular}{|c|c|c|c|c|c|c|c|c|c|c|c|c|c|c|c|c|}
\hline & \multicolumn{4}{|c|}{ Comrade network } & \multicolumn{4}{|c|}{ Enemy network } & \multicolumn{4}{|c|}{ Militant-to-topic network } & \multicolumn{4}{|c|}{ Discursive network } \\
\hline & \multicolumn{4}{|c|}{ Stage } & \multicolumn{4}{|c|}{ Stage } & \multicolumn{4}{|c|}{ Stage } & \multicolumn{4}{|c|}{ Stage } \\
\hline & 1 & 2 & 3 & 4 & 1 & 2 & 3 & 4 & 1 & 2 & 3 & 4 & 1 & 2 & 3 & 4 \\
\hline$N$ nodes & 55 & 54 & 53 & 48 & 55 & 54 & 53 & 48 & - & - & - & - & 10 & 10 & 10 & 10 \\
\hline Density & 0.02 & 0.02 & 0.03 & 0.03 & 0.01 & 0.01 & 0.01 & 0.01 & 0.32 & 0.24 & 0.49 & 0.53 & 0.4 & 0.29 & 0.2 & 0.42 \\
\hline Avg. degree & 0.82 & 1.24 & 1.62 & 1.66 & 0.27 & 0.46 & 0.75 & 0.58 & 3.17 & 2.39 & 4.9 & 5.32 & 3.6 & 2.6 & 1.8 & 3.8 \\
\hline$N$ ties & 45 & 68 & 89 & 91 & 15 & 24 & 41 & 32 & 95 & 79 & 142 & 165 & 18 & 13 & 9 & 19 \\
\hline
\end{tabular}

Notes: Nodes in the Comrade and Enemy networks are individual militants. Nodes in the Discursive network are topics.

Table 3: Change Statistics during Periods

\begin{tabular}{|c|c|c|c|c|c|c|c|c|c|c|c|c|}
\hline \multirow[t]{3}{*}{ Tie change } & \multicolumn{3}{|c|}{ Comrade network } & \multicolumn{3}{|c|}{ Enemy network } & \multicolumn{3}{|c|}{ Militant-to-topic network } & \multicolumn{3}{|c|}{ Discursive network } \\
\hline & \multicolumn{3}{|c|}{ Period } & \multicolumn{3}{|c|}{ Period } & \multicolumn{3}{|c|}{ Period } & \multicolumn{3}{|c|}{ Period } \\
\hline & $1 \rightarrow 2$ & $2 \rightarrow 3$ & $3 \rightarrow 4$ & $1 \rightarrow 2$ & $2 \rightarrow 3$ & $3 \rightarrow 4$ & $1 \rightarrow 2$ & $2 \rightarrow 3$ & $3 \rightarrow 4$ & $1 \rightarrow 2$ & $2 \rightarrow 3$ & $3 \rightarrow 4$ \\
\hline $0 \rightarrow 0$ & 2,885 & 2,858 & 2,861 & 2,940 & 2,923 & 2,927 & 164 & 123 & 60 & 24 & 29 & 24 \\
\hline $0 \rightarrow 1$ & 40 & 44 & 20 & 15 & 22 & 2 & 21 & 93 & 52 & 3 & 3 & 12 \\
\hline $1 \rightarrow 0$ & 17 & 23 & 18 & 5 & 6 & 11 & 44 & 21 & 41 & 8 & 7 & 2 \\
\hline $1 \rightarrow 1$ & 28 & 45 & 71 & 10 & 19 & 30 & 41 & 43 & 77 & 10 & 6 & 7 \\
\hline Distance & 47 & 65 & 25 & 19 & 28 & 8 & 65 & 114 & 93 & 22 & 20 & 28 \\
\hline Jaccard Coeff. & 0.33 & 0.4 & 0.65 & 0.33 & 0.4 & 0.7 & 0.39 & 0.27 & 0.45 & 0.48 & 0.36 & 0.33 \\
\hline
\end{tabular}

Notes: The "Tie change" column indicates whether no-ties (0) between nodes are remaining absent or changing into ties (1), and whether ties are dissolving into no-ties (0) or remaining (1). The Jaccard coefficient provides information on the stability of the network across stages by measuring the Jaccard distance between successive networks. For more information, see Ripley et al. 2019. 
Table 4: Hypothesized Mechanisms

\begin{tabular}{lll}
\hline Hypothesis & SAOM effect description & Time $t_{m}$ \\
\hline $\begin{array}{l}\text { The more militants are linked } \\
\text { to the same cultural elements, } \\
\text { the more likely they are to form } \\
\text { comrade ties, as long as they } \\
\text { hold the same level of power. }\end{array}$ & $\begin{array}{l}\text { Effect of agreement in militant- } \\
\text { to-topic network on comrade } \\
\text { ing the same level of power }\end{array}$ \\
$\begin{array}{l}\text { The more militants receive } \\
\text { comrade ties, the more likely } \\
\text { they are to establish new con- } \\
\text { nections to cultural elements }\end{array}$ & $\begin{array}{l}\text { Effect of indegree in comrade } \\
\text { network on activity in militant- }\end{array}$
\end{tabular}

Notes: Circles are individuals and rectangles are topics. Solid lines represent ties between individuals; dashed lines reflect relationships from militants to topics. Effect descriptions taken from Ripley et al. 2019.

\subsubsection{Covariate effects}

The methodological scholarship on SAOMs identifies basic structural effects that should be included in models (see Ripley et al. 2019). These effects account for how existing network structure can influence the subsequent formation (or dissolution or maintenance) of ties. For example, the tendency for people to reciprocate friendly relations should motivate researchers to include an effect for reciprocity. Other common structural effects capture transitivity, indegree popularity (i.e., popular people tend to receive more ties), and outdegree activity (i.e., individuals who have formed many ties tend to continue to form new ties). We include the commonly recommended structural effects in our models.

We also include effects modeling the influence of militants' personal attributes. The selection of effects and attributes is based on the case-specific literature and the conflict studies scholarship. First, because observers have long noted that militants in Afghanistan were typically friendly with co-ethnics and members of the same militant organization (Rubin 2002; Barfield 2010), we model how shared ethnic group and organizational membership influence the comrade network. Second, drawing on Gould's (2003) and Christia's (2012) insights that power helps determine alliances and animosity, we model the effect of relative power on the formation or maintenance of comrade ties. Finally, because recent research on conflict dynamics has found evidence that ideological similarity encourages the presence of alliances (Gade et al. 2019a), we include a term modeling how militants' structural equivalence with regards to discursive topics influences their comrade ties (see also Vaisey and Lizardo 2010; Lewis et al. 2012; Edelmann and Vaisey 2014). Note that the last two covariates function as the main effects for the first explanatory effect. 


\subsubsection{Modeling sequence}

We estimate three models. ${ }^{15}$ The first uses data only from the two social networks. This provides a "social world baseline", enabling insights into how incorporating multilevel sociocultural mechanisms into our analyses could alter insights from a solely social rendering of conflict dynamics. Model 1 includes the effects accounting for structural and personal attribute mechanisms operating among militants.

The second model adds the militant-to-topic and discursive networks, along with their structural effects and interdependencies with the social network. This model estimates the effect of militants' incoming comrade ties on the formation or maintenance of ties to discursive topics (referred to as "incoming comrade ties affect activity" in the Results section). This effect corresponds to our second hypothesis. The model also includes effects capturing militants' relative power and their structural equivalence with regards to discursive topics - the main effects of the focal interaction term. The focal interaction term is included in Model 3. This effect, which estimates the interaction of power homophily and structural equivalence with regards to discursive topics (referred to as "agreement along militant-to-topic ties, conditional on relative power" in the Results section), corresponds to our first hypothesis.

After estimating a model, we check the convergence of each parameter using its t-ratio, a comparison of the average deviation of the estimated value from its observed value to the standard deviation of this deviation. The models' overall convergence is measured by a maximum convergence ratio, formulated through the same logic (for details, see Ripley et al. 2019). We assess models' goodness of fit by using the estimated parameters to simulate the transition of networks from a preceding period, then compare unmodeled endogenous features of the simulated networks to the same features in the observed network (Lospinoso 2012; Leifeld and Cranmer 2016).

\section{Results}

Table 5 presents the results of the three models. We first see that all our models have achieved excellent convergence. Each parameter has a t-ratio below 0.1, as advised by the methodological literature, and the overall convergence for each model is 0.15 or less (Ripley et al. 2019). Assessments of goodness of fit show that the estimated models achieved satisfactory fit Appendix (F).

The results of Model 3 offer evidence in support of our first hypothesis. Militants have a greater probability of creating or maintaining a comrade tie to another militant the more they share associations with discursive topics and when they hold the same level of power (i.e., "agreement along militant-to-topic ties, conditional on relative power" in Table $5 ; p<0.05)$. Figure 2 shows how this pattern contributes to militants' establishment of comrade relations across increasing amounts of shared connections to topics, as well

\footnotetext{
${ }^{15}$ We implement our SAOMs using RSiena version 1.2.4 with R 3.3.4 (Ripley et al. 2017). Parameters are estimated using the method of moments approach. We assume the probability of receiving an opportunity to make a tie change is equal for all actors for each period.
} 
as how it depends on the level of power. ${ }^{16}$ Indeed, Figure 2 starkly illustrates how increasing the number of shared connections with topics decreases the likelihood of forming ties of affinity when militants have different levels of power. This evidence underscores the possibility of "situations" (Mische and White 1998): shared cultural elements may be drawing militants together, but differing levels of power make it easier for a more powerful militant to contradict and criticize the weaker one, thereby decreasing the probability of forming comrade ties.

We also find evidence in support of our second hypothesis. Militants have a greater probability of forming connections to topics the more they receive friendly ties from other militants (i.e., "incoming comrade ties affect activity" in Table $5 ; p<0.05)$. The pattern is positive and increases across the dataspace: the more incoming comrade ties there are, the greater the contribution to the objective function.

Model 3 sheds additional light on other aspects of relational conflict dynamics. The effect of relative power indicates that militants in symmetrical power relations are less likely to form comrade ties, supporting Gould's (2003) conclusions that symmetrical power in interpersonal interactions can engender conflict, as well as Christia's (2012) argument that asymmetrical power relations encourage militants to forge alliances (see also Gade et al. 2019a). Militants also tend to form or maintain comrade ties if they belong to the same militant group. The negative density coefficients in each of the networks show that ties are formed relatively infrequently across levels of the socio-semantic system.

Several other findings indicate that both structure endogenous to a single network and interdependencies across networks affect affinity and animosity between militants. For example, militants have a tendency for outdegree activity. The more outgoing comrade or enemy ties a militant has, they more likely he is to create or maintain an outgoing comrade or enemy tie in the future. Militants are also likely to reciprocate comrade ties. With regards to cross-network interdependence, the more enemy ties a militant receives, the more likely he is to receive a comrade tie (i.e., "incoming enemy ties affect popularity" in Table 5). At the same time, militants tend to form these enemy ties with the comrades of their enemies (i.e., "the friend of my enemy is my enemy" in Table 5). These interdependencies suggest that future research analyzing alliances or enmity in conflict settings should jointly consider both kinds of relations. Furthermore, we find that topics tend to bind together through the mechanism of triadic closure. We elaborate on this finding in the Discussion section.

Finally, a comparison of Models 1 and 3 offers provisional insights into how multilevel sociocultural mechanisms advance our understanding of conflict dynamics. These insights, to be clear, are merely suggestive since the coefficients are not clearly comparable across models (Mood 2010). With that in mind, we point to two results. First, including data on the social-to-semantic and semantic levels, as Model 3 does, generates mostly minor changes to the results obtained with Model 1, which is the model that draws solely from data on interpersonal relationships, or what we have termed the "social level baseline" model. All but one of the

\footnotetext{
${ }^{16}$ Figure 2 indicates that having a few shared connections to topics, regardless of relative power, negatively contributes to the objective function. Probing the reasons for this is beyond the scope of this study, so we encourage future research to explore why having a small number of shared cultural associations might decrease the likelihood of forming ties of affinity.
} 
Table 5: Results of Stochastic Actor-Oriented Models

\begin{tabular}{|c|c|c|c|}
\hline Dep. Var.: Formation or maintenance of tie & Model 1 & Model 2 & Model 3 \\
\hline \multicolumn{4}{|l|}{ Comrade network (social level) } \\
\hline Rate parameter for Period 1 & $\begin{array}{c}2.454 \\
(0.524)\end{array}$ & $\begin{array}{c}2.448 \\
(0.494)\end{array}$ & $\begin{array}{c}2.293 \\
(0.496)\end{array}$ \\
\hline Rate parameter for Period 2 & $\begin{array}{c}2.463 \\
(0.394)\end{array}$ & $\begin{array}{c}2.459 \\
(0.391)\end{array}$ & $\begin{array}{c}2.509 \\
(0.396)\end{array}$ \\
\hline Rate parameter for Period 3 & $\begin{array}{c}0.826 \\
(0.180)\end{array}$ & $\begin{array}{c}0.821 \\
(0.180)\end{array}$ & $\begin{array}{c}0.819 \\
(0.174)\end{array}$ \\
\hline Density & $\begin{array}{c}-7.850^{* * *} \\
(0.977)\end{array}$ & $\begin{array}{c}-7.943^{* * *} \\
(1.088)\end{array}$ & $\begin{array}{c}-7.534^{* * *} \\
(1.007)\end{array}$ \\
\hline Reciprocity & $\begin{array}{c}2.407^{* * *} \\
(0.370)\end{array}$ & $\begin{array}{c}2.402^{* * * *} \\
(0.366)\end{array}$ & $\begin{array}{c}2.494^{* * *} \\
(0.389)\end{array}$ \\
\hline Transitive closure & $\begin{array}{c}-0.041 \\
(0.499)\end{array}$ & $\begin{array}{c}-0.056 \\
(0.479)\end{array}$ & $\begin{array}{l}-0.009 \\
(0.503)\end{array}$ \\
\hline Cyclical closure & $\begin{array}{l}-0.377 \\
(0.392)\end{array}$ & $\begin{array}{l}-0.348 \\
(0.398)\end{array}$ & $\begin{array}{l}-0.229 \\
(0.429)\end{array}$ \\
\hline Popularity of alter & $\begin{array}{l}0.547^{*} \\
(0.263)\end{array}$ & $\begin{array}{c}0.486 \\
(0.274)\end{array}$ & $\begin{array}{c}0.575 \\
(0.297)\end{array}$ \\
\hline Outdegree activity & $\begin{array}{c}2.346^{* * *} \\
(0.490)\end{array}$ & $\begin{array}{c}2.381^{* * *} \\
(0.557)\end{array}$ & $\begin{array}{c}2.461^{* * *} \\
(0.496)\end{array}$ \\
\hline Same ethnicity & $\begin{array}{l}-0.128 \\
(0.520)\end{array}$ & $\begin{array}{l}-0.198 \\
(0.526)\end{array}$ & $\begin{array}{r}-0.120 \\
(0.609)\end{array}$ \\
\hline Same militant group & $\begin{array}{c}1.075^{* * *} \\
(0.282)\end{array}$ & $\begin{array}{c}0.932^{* *} \\
(0.317)\end{array}$ & $\begin{array}{c}0.952^{* *} \\
(0.336)\end{array}$ \\
\hline Same level of power & $\begin{array}{c}0.099 \\
(0.225)\end{array}$ & $\begin{array}{c}0.082 \\
(0.231)\end{array}$ & $\begin{array}{c}-1.687^{*} \\
(0.823)\end{array}$ \\
\hline Incoming enemy ties affect popularity & $\begin{array}{c}0.623^{* *} \\
(0.191)\end{array}$ & $\begin{array}{c}0.644^{* * *} \\
(0.192)\end{array}$ & $\begin{array}{c}0.656^{* * *} \\
(0.233)\end{array}$ \\
\hline Outgoing enemy ties affect activity & $\begin{array}{l}-0.220 \\
(0.352)\end{array}$ & $\begin{array}{l}-0.229 \\
(0.360)\end{array}$ & $\begin{array}{l}-0.274 \\
(0.392)\end{array}$ \\
\hline "The enemy of my enemy is my friend" & $\begin{array}{l}-1.862 \\
(1.191)\end{array}$ & $\begin{array}{l}-1.967 \\
(1.314)\end{array}$ & $\begin{array}{l}-1.530 \\
(1.344)\end{array}$ \\
\hline $\begin{array}{l}\text { "The enemy of someone who made me their } \\
\text { enemy is my friend" }\end{array}$ & $\begin{array}{l}-2.948 \\
(2.418)\end{array}$ & $\begin{array}{l}-3.112 \\
(2.523)\end{array}$ & $\begin{array}{l}-2.270 \\
(1.984)\end{array}$ \\
\hline Agreement along militant-to-topic ties & & $\begin{array}{c}0.151 \\
(0.164)\end{array}$ & $\begin{array}{l}-0.540 \\
(0.377)\end{array}$ \\
\hline $\begin{array}{l}\text { Agreement along militant-to-topic ties, } \\
\text { conditional on relative power }\end{array}$ & & & $\begin{array}{l}1.147^{*} \\
(0.474)\end{array}$ \\
\hline \multicolumn{4}{|l|}{ Enemy network (social level) } \\
\hline Rate parameter for Period 1 & $\begin{array}{l}1.316 \\
(0.511)\end{array}$ & $\begin{array}{c}1.334 \\
(0.528)\end{array}$ & $\begin{array}{l}1.308 \\
(0.488)\end{array}$ \\
\hline Rate parameter for Period 2 & $\begin{array}{l}1.171 \\
(0.309)\end{array}$ & $\begin{array}{c}1.163 \\
(0.282)\end{array}$ & $\begin{array}{l}1.164 \\
(0.300)\end{array}$ \\
\hline Rate parameter for Period 3 & $\begin{array}{c}0.267 \\
(0.100)\end{array}$ & $\begin{array}{c}0.268 \\
(0.099)\end{array}$ & $\begin{array}{c}0.265 \\
(0.096)\end{array}$ \\
\hline Density & $\begin{array}{l}-9.391^{* * *} \\
(2.605)\end{array}$ & $\begin{array}{l}-9.502^{* * *} \\
(2.679)\end{array}$ & $\begin{array}{c}-9.520^{* *} \\
(2.946)\end{array}$ \\
\hline Reciprocity & $\begin{array}{c}1.149 \\
(1.427)\end{array}$ & $\begin{array}{l}1.170 \\
(1.511)\end{array}$ & $\begin{array}{c}1.150 \\
(1.464)\end{array}$ \\
\hline Popularity of alter & $\begin{array}{c}0.784 \\
(0.425)\end{array}$ & $\begin{array}{c}0.785 \\
(0.453)\end{array}$ & $\begin{array}{c}0.774 \\
(0.447)\end{array}$ \\
\hline Outdegree activity & $\begin{array}{l}1.968^{*} \\
(0.791)\end{array}$ & $\begin{array}{l}1.978^{*} \\
(0.809)\end{array}$ & $\begin{array}{l}1.940^{*} \\
(0.858)\end{array}$ \\
\hline Incoming comrade ties affect popularity & $\begin{array}{c}0.716 \\
(0.391)\end{array}$ & $\begin{array}{c}0.715 \\
(0.401)\end{array}$ & $\begin{array}{c}0.726 \\
(0.402)\end{array}$ \\
\hline Outgoing comrade ties affect activity & $\begin{array}{l}-2.394 \\
(1.739)\end{array}$ & $\begin{array}{l}-2.468 \\
(1.826)\end{array}$ & $\begin{array}{l}-2.537 \\
(2.045)\end{array}$ \\
\hline "The enemy of my friend is my enemy" & $\begin{array}{c}3.468 \\
(2.498)\end{array}$ & $\begin{array}{c}3.595 \\
(2.606)\end{array}$ & $\begin{array}{c}3.716 \\
(2.923)\end{array}$ \\
\hline "The friend of my enemy is my enemy" & $\begin{array}{l}1.004^{*} \\
(0.505)\end{array}$ & $\begin{array}{l}1.025^{*} \\
(0.509)\end{array}$ & $\begin{array}{l}1.075^{*} \\
(0.530)\end{array}$ \\
\hline
\end{tabular}


Table 5, continued...

\begin{tabular}{|c|c|c|c|}
\hline & Model 1 & Model 2 & Model 3 \\
\hline \multicolumn{4}{|l|}{ Militant-to-topic network } \\
\hline Rate parameter for Period 1 & & $\begin{array}{c}3.826 \\
(0.616)\end{array}$ & $\begin{array}{c}3.806 \\
(0.609)\end{array}$ \\
\hline Rate parameter for Period 2 & & $\begin{array}{l}10.862 \\
(2.524)\end{array}$ & $\begin{array}{l}10.779 \\
(2.864)\end{array}$ \\
\hline Rate parameter for Period 3 & & $\begin{array}{c}9.221 \\
(1.846)\end{array}$ & $\begin{array}{c}9.292 \\
(1.952)\end{array}$ \\
\hline Density & & $\begin{array}{l}-0.108 \\
(0.060)\end{array}$ & $\begin{array}{r}-0.104 \\
(0.060)\end{array}$ \\
\hline Incoming comrade ties affect activity & & $\begin{array}{l}0.254^{*} \\
(0.116)\end{array}$ & $\begin{array}{c}0.253^{*} \\
(0.108)\end{array}$ \\
\hline Outgoing comrade ties affect activity & & $\begin{array}{c}0.014 \\
(0.071)\end{array}$ & $\begin{array}{c}0.018 \\
(0.069)\end{array}$ \\
\hline \multicolumn{4}{|l|}{ Discursive network } \\
\hline Rate parameter for Period 1 & & $\begin{array}{c}2.064 \\
(0.936)\end{array}$ & $\begin{array}{c}2.052 \\
(0.915)\end{array}$ \\
\hline Rate parameter for Period 2 & & $\begin{array}{c}2.72 \\
(1.035)\end{array}$ & $\begin{array}{c}2.184 \\
(1.134)\end{array}$ \\
\hline Rate parameter for Period 3 & & $\begin{array}{c}4.193 \\
(2.881)\end{array}$ & $\begin{array}{c}4.165 \\
(3.000)\end{array}$ \\
\hline Density & & $\begin{array}{l}-1.368 \\
(1.695)\end{array}$ & $\begin{array}{c}-1.329 \\
(1.717)\end{array}$ \\
\hline Triadic closure & & $\begin{array}{l}1.241^{*} \\
(0.547)\end{array}$ & $\begin{array}{l}1.230^{*} \\
(0.548)\end{array}$ \\
\hline Four-cycles & & $\begin{array}{l}-0.339 \\
(0.201)\end{array}$ & $\begin{array}{r}-0.334 \\
(0.204)\end{array}$ \\
\hline Popularity of alter & & $\begin{array}{c}0.188 \\
(1.054) \\
\end{array}$ & $\begin{array}{c}0.167 \\
(1.072)\end{array}$ \\
\hline Convergence $t$ ratios all less than. & 0.05 & 0.05 & 0.06 \\
\hline Overall maximum convergence ratio & 0.11 & 0.13 & 0.15 \\
\hline
\end{tabular}

Notes: Table shows log-odds estimates, with standard errors in parentheses. Significance levels of rate parameters are not provided because testing that they are zero is meaningless, as zero would indicate no change between successive waves. For both models, social level node $N=55$ and discursive level node $N$ $=10$. The transitive and cyclical closure effects are defined using geometrically weighted edgewise shared partners. The alter popularity and activity effects are square-root transformed, thus helping to model the diminishing returns of additional ties. ${ }^{*} p<0.05 ;{ }^{* *} p<0.01 ;{ }^{* * *} p<0.001$

social level coefficients maintain their direction and sign when estimating the more complex socio-semantic model. Second, the one change is an effect endogenous to the comrade network (i.e., "popularity of alter" in Table 5 losing its statistical significance at the 0.05 threshold. This suggests that when multilevel sociocultural mechanisms of tie formation are omitted, the strength of endogenous network mechanisms may be biased upwards.

In our view, the minor changes resulting from the incorporation of socio-semantic data do not undermine the usefulness of the multilevel socio-semantic approach. To the contrary, there are good theoretical reasons for using a multilevel graph to analyze how social process and cultural dynamics are interwoven. In addition, there is an empirical payoff from using this approach: we can detail the extent to which not only friendly interpersonal relationships result from connections to culture, but also how these connections to culture are 
Figure 2: Effect of Shared Ties to Topics and Relative Power on Comradeship

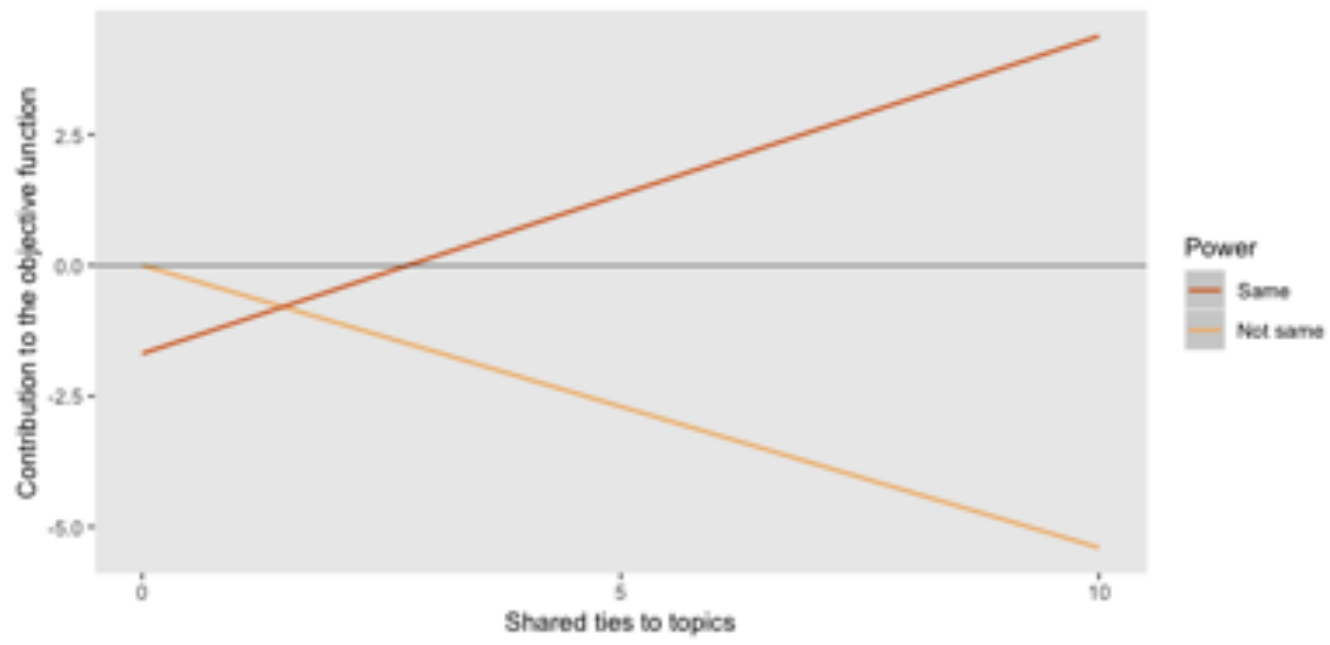

Notes: Figure shows how the effect of agreement on ties to topics conditional on relative power contributes to the objective function for forming or maintaining comrade ties. The contribution is depicted across different values of shared ties and having the same level of power or not.

themselves created. The simple lesson of the comparison, it appears, is that it is possible to gain useful initial insight into a social world shaped, in part, by sociocultural mechanisms even when sociocultural data are omitted.

\section{Discussion}

Our analysis shows how social and cultural dynamics can be entwined during periods of sociopolitical conflict. We find evidence of the cultural domain affecting social relationships: militants' probability of becoming (or remaining) comrades increases as they are connected to more of the same discursive topics, but only if they share similar levels of power. We also find evidence that social relationships shape militants' connections to the cultural domain. Receiving more comrade ties encourages militants to establish new connections with discursive topics.

The findings underscore the role of power and inequity in sociocultural processes. Shared culture and power interact, we argue, because connections to the same discursive elements draw militants into interpersonal encounters, which can engender narratives of affinity when militants hold in abeyance conflicting or discordant perspectives or self-understandings (Mische and White 1998; White 2008). The suspension of interpersonal discordance is more likely among equally powerful militants. In contrast, when one is more powerful than another, he would feel freer to express criticisms and disagreements during the encounter.

Our results also shed light on how endogenous network structure and interdependencies between kinds of relations can help generate interpersonal affinity and animosity. For example, as indicated by the "outdegree activity" effect, the more comradeships or enemies a militant has established in the past, the more likely he 
is to create or maintain an outgoing comrade or enemy tie. In addition, the more militants receive enemy ties, the more they tend to form comrade ties. Militants also typically become enemies with the enemies of their friends.

\subsection{Contributions and future research}

The analysis suggests that fostering shared connections to culture can help promote comradeship during conflict. This insight has important implications for building peace in war-torn contexts, since unity among militants militates against longer conflicts and conflict recurrence (Cunningham 2006; Blattman and Miguel 2010; Cederman and Vogt 2017; Gade et al. 2019a). One specific lesson offered by our study is that culture can expand the opportunities for creating comradeship. Without shared culture, evidence suggests that comradeship is likely only under conditions of asymmetrical power between militants (Christia 2012). With shared culture, comrade ties become likely under the alternative condition of symmetrical power. Another lesson is that the generation of comrade relations can be self-sustaining. For instance, if conflict mediators help build some comrade ties, these ties can lead to new connections to cultural elements, which can result in more comrade ties being established, and so on. We encourage future research to further explore how sociocultural mechanisms might endogenously promote interpersonal affinity during times of conflict.

More broadly, our study helps incorporate culture into conflict studies beyond the current focus on ideology (e.g., Gutiérrez Sanín and Wood 2014; Gade et al. 2019a; Maynard 2019). We suggest that future conflict studies research considers culture as a coherent and changing set of connected concepts, discursive topics, and ideas - with or without grievances, objectives, or plans - that derive their meanings and influence both from their associations with one another, as well as their links to the social realm. Doing so can help make sense of complex conflicts, such as when combatants establish webs of alliances and rivalries within and across ideological lines.

Our study also points to possible developments in formal cultural sociology and, more specifically, sociosemantic network research. First, by highlighting the understudied case of sociopolitical conflict, we increase the opportunities for specifying the bounds of previously identified sociocultural mechanisms - a goal of the formal study of culture (Edelmann and Mohr 2018). For example, we may ask whether the tendency for actors to culturally differentiate from one another once a cultural ecosystem hits "carrying capacity" (Kaufman 2004; Askin and Mauskapf 2017; Godart and Galunic 2019) also occurs in conflict settings, where differentiation may in fact result in new enemies.

In addition, the article analytically specifies and empirically evaluates Mische and White's (1998) insight into how social ties emerge from interpersonal encounters - encounters that may become contentious. We do so by defining a mechanism capturing the influence of individuals' structural equivalence with regards to cultural elements, conditional on their relative power. Further research on this mechanism could investigate why we observed a negative contribution to the objective function when individuals only shared a few connections to topics, despite having the same level of power. Future work could also help determine its 
general applicability. Indeed, the evidence we find of power and relations of animosity affecting social and cultural ties suggests that studies of sociocultural processes, such as in the socio-semantic network and "cultural tastes" literatures (e.g., Lizardo 2006; Lewis et al. 2012; Lewis and Kaufman 2018), could uncover new mechanisms by considering power and enmity.

Furthermore, our results offer a glimpse of how cultural space evolves endogenously. Cultural elements are structurally embedded among other elements (Somers 1995; Carley 1997; Mohr 1998; Bearman and Stovel 2000; Somers 2008; Lee and Martin 2015), and this embeddedness can be weighed through connections between elements (Moran 2005; Godart and Galunic 2019). Following these insights, we developed a methodological approach that can analyze how cultural elements' embeddedness changes over time. For instance, we find evidence that when cultural elements are directly unconnected but associated with a same third element, they tend to become associated in the future (i.e., "triadic closure" in Table 5). Theorizing the endogenous process of triadic closure in cultural space is beyond the scope of our study, but we speculate that the finding reflects the tendency for cultural elements to become increasingly associated as combatants forge dominant narratives over the course of a conflict to make sense of coalescing alliances or conflict resolution (Christia 2012).

Finally, our synthesis of topic models and SAOMs offers socio-semantic network scholars some key analytical researchers. First, they can define cultural space without using presumed categories. Second, this definition can be validated, falsified, and reproduced. Third, researchers can analyze mechanisms of network change operating within and across the levels of a multiplex and multilevel graph over time. That is, not only do "text-SAOMs" incorporate continuous time into the modeling process, but their focus on actors' tie-formation or tie-dissolution in the context of social relationships and cultural space offers a way to quantitatively examine interdependencies of agency and structure (Emirbayer 1997; Hollway et al. 2017; Lewis and Kaufman 2018; Edelmann and Mohr 2018).

We conclude this section with brief comments on how our analytical approach helps socio-semantic network scholars contribute to the methodological development of longitudinal network modeling and computational textual analysis. Namely, socio-semantic network scholars can use our approach to broaden existing SAOM analyses, which typically focus on the social domain (e.g., person-to-person, organization-toorganization, or person-and-organization). This effort can be both theoretical and empirical. For example, which mechanisms, according to socio-semantic network theory and evidence, should we expect to operate in the semantic portions of longitudinal multilevel socio-semantic graphs?

Turning to computational textual research, this body of work increasingly recognizes cultural sociology's insight that semantic categories are not only dependent on the static distribution of words across documents, but also on the previous realizations of the semantic space (Rule et al. 2015). As a result, recently developed topic models are beginning to examine temporal dependency by tracing content and topic attributes across time (Gerowa et al. 2018). The approach we develop here offers a complementary method. It enables the analysis of dynamic structural dependencies, thereby emphasizing how relationships between semantic 
elements can influence future elements. Moreover, socio-semantic scholars using our approach could explore how to interpret the meaning of output from computational models by placing it in its sociocultural context (Fushe and Mützel 2011). This can be done by considering, for example, how topics are linked to social entities or by incorporating the social links as covariates in STMs.

\subsection{Limitations}

In addition to the specific data and methodological challenges discussed throughout the article, our analytical approach has a few general limitations. Among these is the loss of information. For example, we collapse several "positive" types of ties into a general "comrade" tie. We do so, in part, because we lack an explicit theory for how or why, say, an "ally" connection would operate differently than a "friend" connection. In addition, by including temporal covariates in our topic model, we fix the content, or nodes, of the discursive network over time. While this limits our insights into the full dynamism of the cultural space, it does allow us to, first, conceptualize this space as a general discourse, and, second, study how the elements of this discourse have changing associations over time.

These limitations underscore that our analytical approach is an initial formulation; it offers opportunities for further development. Future research could investigate how different kinds of comrade ties differ (if at all) or use these ties to construct a scale of tie strength. Other work could model changes in the content of the semantic level, perhaps by incorporating dynamic topic models (Blei and Lafferty 2006; Gerowa et al. 2018). After all, the concepts embedded within cultural space do transform over time (Rule et al. 2015). Additional developments can make use of SAOMs' ability to incorporate exogenous information about semantic nodes. For example, researchers can measure the sentiment of topics, build semantic-node variables for sentiment, and use basic SAOM specifications to analyze how the sentiment of semantic concepts influences network evolution.

In addition, researchers should consider whether topic models are appropriate for their cultural data. Short texts, for example, may not be suited to topic models (Bail 2016). In these cases, other clustering techniques may provide better insight (Nelson 2017). However, our overall argument regarding the analytical approach remains the same: computational methods for analyzing text can help create representations of semantic space over time - especially when researchers are drawing on large bodies of textual data - and these representations can then be analyzed using SAOMs.

\section{Conclusion}

How do sociocultural dynamics shape conflict? We find that when comparatively powerful militants are increasingly connected to the same discursive topics, they have a greater probability of becoming comrades. In addition, militants' tendency to form connections to discursive topics increases the more other militants identify them as comrades. Put differently, shared culture interacts with power to engender comradeship in 
settings of contention, and individuals' links to culture are themselves a result of comrade relations. A key implication of this process is that, during conflict, comradeship can be encouraged through the promotion of a shared discursive space. For cultural sociologists, our findings focus attention on the role of relative power in shaping the mutual constitution of social and cultural worlds.

We analyze and explain the interdependency of culture, social relations, and conflict by drawing together insights from two literatures: conflict studies and cultural sociology. We apply these insights in a study of militants' relationships and culture over two decades of conflict in one Afghan province, Balkh. The relationships are captured by a longitudinal multilevel graph comprising networks of affinity and animosity among militants, discursive topics, and militant-topic connections. Our analysis of this socio-semantic graph employs a novel approach that synthesizes computational textual analysis and stochastic actor-oriented models ("text-SAOMs"). This approach enables us to define cultural space from a large textual dataset, model continuous-time dynamics, and identify mechanisms operating within and across levels, as well as over time. Doing so provides new possibilities for research on socio-semantic networks and sociocultural dynamics 


\section{References}

Ai, Chunrong and Edward Norton. 2003. "Interaction Terms in Logit and Probit Models." Economics Letters 80:123-129.

Archer, Margaret. 1998. Culture and Agency: The Place of Culture in Social Theory. New York: Cambridge University Press.

Askin, Noah and Michael Mauskapf. 2017. "What Makes Popular Culture Popular? Product Features and Optimal Differentiation in Music." American Sociological Review 82:910-944.

Bail, Christopher. 2016. "Combining Natural Language Processing and Network Analysis to Examine How Advocacy Organizations Stimulate Conversation on Social Media." Proceedings of the National Academy of Sciences www.pnas.org/cgi/doi/10.1073/pnas.1607151113.

Barfield, Thomas. 2010. Afghanistan: A Cultural and Political History. Princeton, NJ: Princeton University Press.

Basov, Nikita. 2018. "Socio-Material Network Analysis: A Mixed Method Study of Five European Artistic Collectives." Social Networks 54:179-195.

Basov, Nikita. 2019. "The Ambivalence of Cultural Homophily: Field Positions, Semantic Similarities, and Social Network Ties in Creative Collectives." Poetics DOI:10.1016/j.poetic.2019.02.004.

Basov, Nikita and Julia Brennecke. 2017. Structure, Content and Meaning of Organizational Networks, chapter Duality Beyond Dyads: Multiplex Patterning of Social Ties and Cultural Meanings, pp. 82-112. ISBN: 978-1-78714-434-7: emeraldinsight.

Basov, Nikita, Ju-Sung Lee, and Artem Antoniuk. 2017. Complex Networks \& Their Applications V, chapter Social Networks and Construction of Culture: A Socio-Semantic Analysis of Art Groups, pp. 785-796. Cham, Switzerland: Springer.

Bearman, Peter. 1993. Relations into Rhetorics: Local Elite State Structure in Norfolk, England, 1540-1640. New Brunswick, NJ: Rutgers University Press.

Bearman, Peter. 2003. Collision of Wills, chapter Forward, pp. ix-xvi. Chicago: University of Chicago Press.

Bearman, Peter and Katherine Stovel. 2000. "Becoming a Nazi: A Model for Narrative Networks." Poetics 27:69-90.

Benjamin, Daniel and Steven Simon. 2002. The Age of Sacred Terror. New York: Random House.

Berman, Eli. 2009. Radical, Religious, and Violent: The New Economics of Terrorism. Cambridge: MIT Press.

Bernard, H. Russell, Peter Killworth, and Lee Sailer. 1979/1980. "Informant Accuracy in Social Network Data IV: A Comparison of Clique-Level Structure in Behavioral and Cognitive Network Data." Social Networks 2:191-218.

Blattman, Christopher and Edward Miguel. 2010. "Civil War." Journal of Economic Literature 48:3-57.

Blei, David and John Lafferty. 2006. "Dynamic Topic Models." Proceedings of the 23rd international Conference on Machine Learning pp. 113-120.

Blei, David, Andrew Ng, and Michael Jordan. 2003. "Latent Dirichlet Allocation." Journal of Machine Learning Research 3:993-1022.

Block, Per, Johan Koskinen, James Hollway, Christian Steglich, and Christoph Stadtfeld. 2018. "Change We can Believe In: Comparing Longitudinal Network Models on Consistency, Interpretability, and Predictive Power." Social Networks 52:189-191. 
Block, Per, Christoph Stadtfeld, and Tom Snijders. 2016. "Forms of Dependence: Comparing SAOMs and ERGMs From Basic Principles." Sociological Methods \& Research DOI: 10.1177/0049124116672680:XX.

Brandes, Ulrik, Garry Robins, Ann McCranie, and Stanely Wasserman. 2013. "What Is Network Science?" Network Science 1:1-15.

Breiger, Ronald and Kyle Puetz. 2015. International Encyclopedia of Social \&3 Behavioral Sciences, chapter Culture and Networks, pp. 557-562. New York: Elsevier, 2 edition.

Carley, Kathleen. 1997. "Extracting Team Mental Models Through Textual Analysis." Journal of Organizational Behavior 18:533-538.

Cederman, Lars-Erik, Kristian Skrede Gleditsch, and Halvard Buhaug. 2013. Inequality, Grievances, and Civil War. New York: Cambridge University Press.

Cederman, Lars-Erik and Manuel Vogt. 2017. "Dynamics and Logics of Civil War." Journal of Conflict Resolution 61:1992-2016.

Christia, Fotini. 2012. Alliance Formation in Civil Wars. New York: Cambridge University Press.

Collier, Paul and Anke Hoeffler. 2004. "Greed and Grievance in Civil War." Oxford Economic Papers $56: 563-595$.

Crandall, David, Dan Cosley, Daniel Huttenlocher, Jon Kleinberg, and Suri Siddharth. 2008. "Feedback Effects between Similarity and Social Influence in Online Communities." KDD '08 14th ACM SIGKDD International Conference on Knowledge Discovery and Data Mining pp. 160-168.

Crost, Benjamin, Joseph Felter, and Patrick Johnston. 2014. "Aid Under Fire: Development Projects and Civil Conflict." American Economic Review 104:1833-1856.

Cunningham, David. 2006. "Veto Players and Civil War Duration." American Journal of Political Science 50:875-892.

Denny, Matthew and Arthur Spirling. 2018. "Text Preprocessing for Unsupervised Learning: Why It Matters, When It Misleads, And What to Do About It." Political Analysis 26:168-189.

DiMaggio, Paul. 2015. "Adapting Computational Text Analysis to Social Science (and Vice Versa)." Big Data \& Society 2:1-5.

Edelmann, Achim and John Mohr. 2018. "Formal Studies of Culture: Issues, Challenges, and Current Trends." Poetics 68:1-9.

Edelmann, Achim and Stephen Vaisey. 2014. "Cultural Resources and Cultural Distinction in Networks." Poetics 46:22-37.

Egami, Naoki, Christian Fong, Justin Grimmer, Margaret Roberts, and Brandon Stewart. 2018. "How to Make Causal Inferences Using Texts." arXiv:1802.02163.

Emirbayer, Mustafa. 1997. "Manifesto for a Relational Sociology." American Journal of Sociology 103:281317.

Erickson, Bonnie. 1996. "Culture, Class, and Connections." American Journal of Sociology 102:217-251.

Farrell, Justin. 2016. "Corporate Funding and Ideological Polarization about Climate Changes." Proceedings of the National Academy of Sciences 113:92-97.

Fearon, James and David Laitin. 2003. "Ethnicity, Insurgency, and Civil War." American Political Science Review 97:1-16.

Ferligoj, Anuška and Valentina Hlebec. 1999. "Evaluation of Social Network Measurement Instruments." Social Networks 21:111-130. 
Freeman, Linton C., Sue C. Freeman, and Alaina G. Michaelson. 1988. "On Human Social Intelligence." Journal of Social and Biological Structures 11:191-218.

Freeman, Linton C. and A. Kimbell Romney. 1987. "Words, Deeds, and Social Structure: A Preliminary Study of the Reliability of Informants." Human Organization 46:330-334.

Freeman, Linton C., A. Kimbell Romney, and Sue C. Freeman. 1987. "Cognitive Structure and Informant Accuracy." American Anthropologist 89:310-325.

Fuhse, Jan, Oscar Stuhler, Jan Riebling, and John Levi Martin. 2019. "Relating Social and Symbolic Relations in Quantitative Text Analysis. A Study of Parliamentary Discourse in the Weimar Republic." Poetics DOI:10.1016/j.poetic.2019.04.004.

Fushe, Jan and Sophie Mützel. 2011. "Tackling Connections, Structure, and Meaning in Networks: Quantitative and Qualitative Methods in Sociological Network Research." Quality \& Quantity 45:1067-1089.

Gade, Emily, Michael Gabbay, Mohammed Hafez, and Zane Kelly. 2019a. "Networks of Cooperation: Rebel Alliances in Fragmented Civil Wars." Journal of Conflict Resolution 63:2071-2097.

Gade, Emily, Mohammed Hafez, and Michael Gabbay. 2019b. "Fratricide in Rebel Movements: A Network Analysis of Syrian Militant Infighting." Journal of Peace Research 56:321-335.

Gerowa, Aaron, Yuening Hu, Jordan Boyd-Graberc, David Blei, and James Evans. 2018. "Measuring Discursive Influence Across Scholarship." Proceedings of the National Academy of Sciences 115:3308-331.

Gerring, John. 2007. Case Study Research: Principles and Practices. New York: Cambridge University Press.

Godart, Frédéric and Charles Galunic. 2019. "Explaining the Popularity of Cultural Elements: Networks, Culture, and the Structural Embeddedness of High Fashion Trends." Organization Science doi.org/10.1287/orsc.2018.1234:1-18.

Goodson, Larry. 2001. Afghanistan's Endless War. Seattle: University of Washington Press.

Gould, Roger. 1995. Insurgent Identities. Chicago: University of Chicago Press.

Gould, Roger. 1996. "Patron-Client Ties, State Centralization, and the Whiskey Rebellion." American Journal of Sociology 102:400-429.

Gould, Roger. 2003. Collision of Wills. Chicago: University of Chicago Press.

Grimmer, Justin and Brandon Stewart. 2013. "Text as Data: The Promise and Pitfalls of Automatic Content Analysis Methods for Political Texts." Political Analysis 21:267-297.

Gurr, Ted. [1970] 2011. Why Men Rebel. Boulder, CO: Paradigm Publishers, 2nd edition.

Gutiérrez Sanín, Francisco and Elisabeth Wood. 2014. "Ideology in Civil War: Instrumental Adoption and Beyond." Journal of Peace Research 51:213-226.

Hechter, Michael, Steven Pfaff, and Patrick Underwood. 2016. "Grievances and the Genesis of Rebellion: Mutiny in the Royal Navy, 1740 to 1820." American Sociological Review 81:165-189.

Hedström, Peter and Petri Ylikoski. 2010. "Causal Mechanisms in the Social Sciences." Annual Review of Sociology 36:49-67.

Hlebec, Valentina and Anuška Ferligoj. 2001. "Respondent Mood and the Instability of Survey Network Measurements." Social Networks 23:125-140.

Hollway, James, Alessandro Lomi, Francesca Pallotti, and Christoph Stadtfeld. 2017. "Multilevel Social Spaces: The Network Dynamics of Organizational Fields." Network Science 5:187-212. 
Huitsing, Gijs, Tom Snijders, Marijtje Van Duijn, and René Veenstra. 2014. "Victims, Bullies, and Their Defenders: A Longitudinal Study of the Coevolution of Positive and Negative Networks." Development and Psychopathology 26:645-659.

Indlekofer, Natalie and Ulrik Brandes. 2013. "Relative Importance of Effects in Stochastic Actor-Oriented Models." Network Science 1:278-304.

Isaacs, Matthew. 2016. "Sacred Violence or Strategic Faith? Disentangling the Relationship between Religion and Violence in Armed Conflict." Journal of Peace Research 53:211-225.

Kaplan, Robert. 1993. Balkan Ghosts. New York: Vintage Books.

Karell, Daniel and Michael Freedman. 2019. "Rhetorics of Radicalism." American Sociological Review $84: 726-753$.

Karell, Daniel and Sebastian Schutte. 2018. "Aid, Exclusion, and the Local Dynamics of Insurgency in Afghanistan." Journal of Peace Research 55:711-725.

Kaufman, Jason. 2004. "Endogenous Explanations in the Sociology of Culture." Annual Review of Sociology $30: 335-357$.

Kawalerowicz, Juta and Michael Biggs. 2015. "Anarchy in the UK: Economic Deprivation, Social Disorganization, and Political Grievances in the London Riot of 2011." Social Forces 94:673-698.

Killworth, Peter D. and H. Russell Bernard. 1979/1980. "Informant Accuracy in Social Network Data III: A Comparison of Triadic Structure in Behavioral and Cognitive Data." Social Networks 2:19-46.

Kim, Jinseok and Jana Diesner. 2015. "The Effect of Data Pre-Processing on Understanding the Evolution of Collaboration Networks." Journal of Informetrics 9:226-236.

Lee, Monica and John Levi Martin. 2015. "Coding, Counting and Cultural Cartography." American Journal of Cultural Sociology 3:1-33.

Leifeld, Philip and Skyler Cranmer. 2016. "A Theoretical and Empirical Comparison of the Temporal Exponential Random Graph Model and the Stochastic Actor-Oriented Model." arXiv: 1506.06696.

Leifeld, Philip, Skyler Cranmer, and Bruce Desmarais. 2017. xergm: Extensions for Exponential Random Graph Models. R package version 1.8.2.

Leszczensky, Lars and Sebastian Pink. 2019. "What Drives Ethnic Homophily? A Relational Approach on How Ethnic Identification Moderates Preferences for Same-Ethnic Friends." American Sociological Review DOI: $10.1177 / 0003122419846849$.

Lewis, Kevin, Marco Gonzalez, and Jason Kaufman. 2012. "Social Selection and Peer Influence in an Online Social Network." Proceedings of the National Academy of Sciences 109:68-72.

Lewis, Kevin and Jason Kaufman. 2018. "The Conversion of Cultural Tastes into Social Network Ties." American Journal of Sociology 123:1684-1742.

Light, Ryan and Colin Odden. 2017. "Managing the Boundaries of Taste: Culture, Valuation, and Computational Social Science." Social Forces 96:877-908.

Lizardo, Omar. 2006. "How Cultural Tastes Shape Personal Networks." American Sociological Review 71:778-807.

Lomi, Alessandro and Christoph Stadtfeld. 2014. "Social Networks and Social Settings: Developing a Coevolutionary View." Kölner Zeitschrift für Soziologie und Sozialpsychologie 68:395-415.

Lospinoso, Joshua. 2012. "Statistical Models for Social Network Dynamics." Doctor of Philosophy thesis Department of Statistics, University of Oxford. 
Lucas, Christopher, Richard Nielsen, Molly Roberts, Brandon Stewart, and Dustin Tingley. 2015. "Computer-Assisted Text Analysis for Comparative Politics." Political Analysis 23:254-277.

Martin, John Levi. 2002. "Power, Authority, and the Constraint of Belief Systems." American Journal of Sociology 107:861-904.

Martin, Mike. 2014. An Intimate War: An Oral History of the Helmand Conflict. New York: Oxford University Press.

Maynard, Jonathan Leader. 2019. "Ideology and Armed Conflict." Journal of Peace Research 56:635-649.

McLean, Paul. 2007. The Art of the Network: Strategic Interaction and Patronage in Renaissance Florence. Durham: Duke University Press.

Mische, Ann. 2007. Partisan Publics: Communication and Contention across Brazilian Youth Activist Networks. Princeton: Princeton University Press.

Mische, Ann. 2011. The SAGE Handbook of Social Network Analysis, chapter Relational Sociology, Culture, and Agency, pp. 80-98. London: SAGE, 2 edition.

Mische, Ann and Harrison White. 1998. "Between Conversation and Situation: Public Switching Dynamics across Network Domains." Social Research 65:695-724.

Mize, Trenton. 2019. "Best Practices for Estimating, Interpreting, and Presenting Nonlinear Interaction Effects." Sociological Science 6:81-117.

Mohr, John. 1998. "Measuring Meaning Structures." Annual Review of Sociology pp. 345-370.

Mohr, John and Petko Bogdanov. 2013. "Topic Models: What They Are and Why They Matter." Poetics 41:545-569.

Mohr, John and Vincent Duquenne. 1997. "The Duality of Culture and Practice: Poverty Relief in New York City, 1888-1917." Theory and Society 26:305-356.

Mood, Carina. 2010. "Logistic Regression: Why We Cannot Do What We Think We Can Do, and What We Can Do About It." European Sociological Review 26:67-82.

Moran, Peter. 2005. "Structural vs. Relational Embeddedness: Social Capital and Managerial Performance." Strategic Management Journal 26:1129-1151.

Mustillo, Sarah, Omar Lizardo, and Rory McVeigh. 2018. "Editors' Comment: A Few Guidelines for Quantitative Submissions." American Sociological Review 83:1281-1283.

Nelson, Laura. 2017. "Computational Grounded Theory: A Methodological Framework." Sociological Methods $\&$ Research DOI:10.1177/0049124117729703:1-40.

Nepal, Mani, Alok Bohara, and Kishore Gawande. 2011. "More Inequality, More Killings: The Maoist Insurgency in Nepal." American Journal of Political Science 55:885-905.

Nerghes, Adina, Ju-Sung Lee, Peter Groenewegen, and Iina Hellsten. 2015. "Mapping Discursive Dynamics of the Financial Crisis: A Structural Perspective of Concept Roles in Semantic Networks." Computational Social Networks 2:DOI 10.1186/s40649-015-0021-8.

Nielsen, Richard. 2017. Deadly Clerics. New York: Cambridge University Press.

Pachucki, Mark and Ronald Breiger. 2010. "Cultural Holes: Beyond Relationality in Social Networks and Culture." Annual Review of Sociology 36:205-224.

Rashid, Ahmed. 2000. Taliban: Islam, Oil, and the New Great Game in Central Asia. New York: I.B. Tauris. 
Ripley, Ruth, Krists Boitmanis, Tom Snijders, and Felix Schoenenberger. 2017. RSiena: Siena - Simulation Investigation for Empirical Network Analysis. R package version 1.2-4/r322.

Ripley, Ruth, Tom Snijders, Zsófia Boda, András Vörös, and Paulina Preciado. 2019. "Manual for RSiena." Oxford: Nuffield College, University of Oxford.

Roberts, Margaret, Brandon Stewart, Dustin Tingley, Christopher Lucas, Jetson Leder-Luis, Shana Kushner Gadarian, Bethany Albertson, and David Rand. 2014. "Structural Topic Models for Open-Ended Survey Responses." American Journal of Political Science 58:1064-1082.

Romney, A. Kimbell and Katherine Faust. 1982. "Predicting the Structure of a Communications Network from Recalled Data." Social Networks 4:285-304.

Roth, Camille. 2013. "Socio-Semantic Frameworks." Advances in Complex Systems 16.

Roth, Camille and Jean-Philippe Cointet. 2010. "Social and Semantic Coevolution in Knowledge Networks." Social Networks 32:16-29.

Rubin, Barnett. 2002. The Fragmentation of Afghanistan. New Haven, CT: Yale University Press, 2nd edition.

Rule, Alix, Jean-Philippe Cointet, and Peter Bearman. 2015. "Lexical Shifts, Substantive Changes, and Continuity in State of the Union Discourse, 1790-2014." Proceedings of the National Academy of Sciences 112:10837-10844.

Schori-Eyal, Noa, Eran Halperin, and Tamar Saguy. 2019. "Intergroup Commonality, Political Ideology, and Tolerance of Enemy Collateral Casualties in Intergroup Conflicts." Journal of Peace Research 56:425-439.

Snijders, Tom. 2001. "The Statistical Evaluation of Social Network Dynamics." Sociological Methodology $31: 361-395$.

Snijders, Tom. 2005. Models and Methods in Social Network Analysis, chapter Models for Longitudinal Network Data, pp. 215-247. New York: Cambridge University Press.

Snijders, Tom and Alessandro Lomi. 2019. "Beyond Homophily: Incorporating Actor Variables in Statistical Network Models." Network Science 7:1-17.

Snijders, Tom, Alessandro Lomi, and Vanina Torlóe. 2013. "A Model for the Multiplex Dynamics of TwoMode and One-Mode Networks, with an Application to Employment Preference, Friendship, and Advice." Social Networks 35:265-276.

Snijders, Tom, Gerhard van de Bunt, and Christian Steglich. 2010. "Introduction to Stochastic Actor-Based Models for Network Dynamics." Social Networks 32:44-60.

Somers, Margaret. 1995. "Narrating and Naturalizing Civil Society and Citizenship Theory: The Place of Political Culture and the Public Sphere." Sociological Theory 13:229-274.

Somers, Margaret. 2008. Genealogies of Citizenship. New York: Cambridge University Press.

Steglich, Christian, Tom Snijders, and Michael Pearson. 2010. "Dynamic Networks and Behavior: Separating Selection from Influence." Sociological Methodology 40:329-393.

Vaisey, Stephen and Omar Lizardo. 2010. "Can Cultural Worldviews Influence Network Composition?" Social Forces 88:1595-1618.

Walter, Barbara. 2017a. "The Extremist's Advantage in Civil Wars." International Security 42:7-39.

Walter, Barbara. 2017b. "The New New Civil Wars." Annual Review of Political Science 20:469-486.

Wang, Peng, Garry Robins, Philippa Pattison, and Emmanuel Lazega. 2013. "Exponential Random Graph Models for Multilevel Networks." Social Networks 1:96-115. 
White, Harrison. 1995. "Network Switchings and Bayesian Forks: Reconstructing the Social and Behavioral Sciences." Social Research 62:1035-1063.

White, Harrison. 2008. Identity and Control. Princeton: Princeton University Press, 2nd edition.

Wilkerson, John and Andreu Casas. 2017. "Large-Scale Computerized Text Analysis in Political Science: Opportunities and Challenges." Annual Review of Political Science 20:529-544.

Zaeef, Abdul Salam. 2010. My Life With the Taliban. New York: Columbia University Press. 


\author{
Online Appendices
}

Sociocultural Mechanisms of Conflict: Combining Topic and Stochastic Actor-Oriented Models in an Analysis of Afghanistan, 1979-2001

Daniel Karell and Michael Freedman

November 2019

Pre-print copy

Published version in Poetics (December 2019)

https://doi.org/10.1016/j.poetic. 2019.101403 


\section{Appendix A Socio-Semantic Networks and the Logic of SAOMs}

Stochastic actor-oriented models (SAOMs) represent graph evolution in continuous time through the simulation of changes to the network between observed waves of the graph. ${ }^{17}$ Longitudinal exponential random graph models (LERGMs) utilize the same approach. So, if both LERGMs and SAOMs incorporate continuous-time dynamics, why do we employ SAOMs in our analytical approach? At first glance, LERGMs appear more appropriate for modeling the semantic level of socio-semantic networks: as models of structurewhat the topology of a network tends to be - rather than of actors, or nodes, initiating ties, LERGMs allow researchers to avoid claiming that semantic elements (and individuals or groups) go through the process of selecting alters that is implied by SAOMs (Snijders 2005).

On the contrary, we argue that the "actor-oriented" nature of SAOMs make them more appropriate than LERGMs for socio-semantic network analyses. Despite common understandings, SAOMs are not actororiented because they assume that nodes select outcomes (whether rationally or not) (Snijders et al. 2010; Ripley et al. 2019: 9-10). Instead, SAOMs are actor-oriented because tie formation, dissolution, and maintenance are based on nodes' context: ties emanate from a node (or not) depending on its other ties, as well as the rest of the network structure. That is, SAOMs, unlike LERGMs, incorporate important information about nodes' embeddedness. They not only consider the topology of nodes' social space, or the networks in which nodes are situated, but also the immediate relations of each node when estimating the probability of a tie-related outcome (Block et al. 2016). This thick dependency better approximates theoretical conceptualizations of semantic levels as spaces saturated with embeddedness. ${ }^{18}$

To explain how SAOMs enable socio-semantic network scholars to better capture the embedding of nodes in network structure, we briefly review an example taken from Block et al. 2016: 17-18. Consider the transition of triad 012 to two possible adjacent states through the addition of one tie, triads $021 \mathrm{C}(\mathrm{a})$ and 021C(b), shown in Figure A.1. In an (L)ERGM that is estimating the effects of density, or outdegree, and reciprocity, the probability of a transition to $021 \mathrm{C}(\mathrm{a})$ and $021 \mathrm{C}(\mathrm{b})$ are equally likely:

$$
\begin{gathered}
p_{\text {ERGM }}(012 \rightarrow 021 \mathrm{C}(\mathrm{a}))=p_{\text {ERGM }}(012 \rightarrow 021 \mathrm{C}(\mathrm{b})) \\
=\frac{1}{6} \times \frac{\exp \left(1 \times \theta_{\text {density }}+0 \times \theta_{\text {reciprocity }}\right)}{1+\exp \left(1 \times \theta_{\text {density }}+0 \times \theta_{\text {reciprocity }}\right)} \\
=\frac{1}{6} \times \frac{\exp \left(\theta_{\text {density }}\right)}{1+\exp \left(\theta_{\text {density }}\right)}
\end{gathered}
$$

\footnotetext{
${ }^{17}$ In contrast, discrete-time models, such as variations of exponential random graph models (ERGMs), like TERGMs, use information from a previous network observation to condition the analysis of a subsequent observation, leaving processes unmodeled. As a result, the estimated parameters of discrete-time models are sensitive to the length of time between observed periods. Continuous-time models lack this sensitivity because changes are modeled over time. For further discussion, see Block et al. 2018.

${ }^{18}$ Somers (1995: 135-36), quoted by Mische (2011), puts it this way: "Concepts cannot be defined on their own as single ontological entities; rather, the meaning of one concept can be deciphered only in terms of its 'place' in relation to the other concepts in its web" (see also Mohr 1998; White 2008).
} 
Figure A.1: Transitions from Triad 012

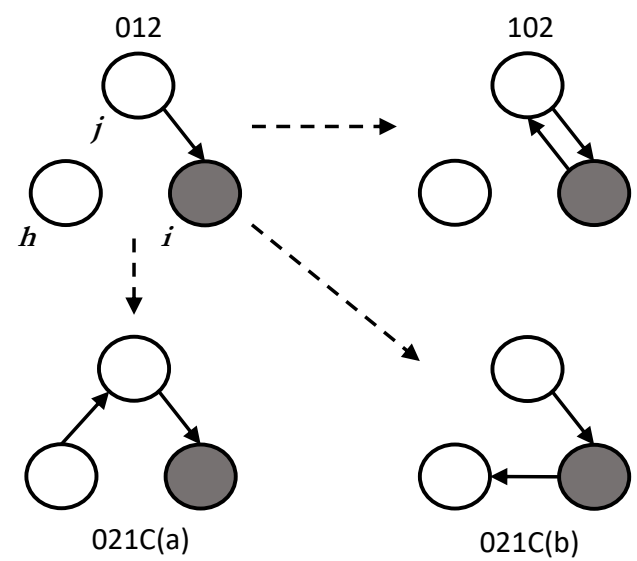

Notes: Figure adapted from Block et al. 2016.

where $\theta$ is a statistical parameter, of type indicated in the subscript. The first fraction in (1) denotes the probability of selecting a triad outcome - there are six possible transitions from 012; we only show three in Figure A.1 - and the reciprocity term drops from the equation because neither $021 \mathrm{C}(\mathrm{a})$ or $021 \mathrm{C}(\mathrm{b})$ have a reciprocated tie.

In contrast, under the SAOM framework, the probability of transitioning to $021 \mathrm{C}(\mathrm{a})$ and $021 \mathrm{C}(\mathrm{b})$ differ:

$$
\begin{gathered}
p_{\mathrm{SAOM}}(012 \rightarrow 021 \mathrm{C}(\mathrm{a}))= \\
\frac{1}{3} \times \frac{\exp \left(\beta_{\text {density }}\right)}{\exp (0)+\exp \left(\beta_{\text {density }}\right)+\exp \left(\beta_{\text {density }}\right)}
\end{gathered}
$$

and

$$
\begin{gathered}
p_{\mathrm{SAOM}}(012 \rightarrow 021 \mathrm{C}(\mathrm{b}))= \\
\frac{1}{3} \times \frac{\exp \left(\beta_{\text {density }}\right)}{\exp (0)+\exp \left(\beta_{\text {density }}\right)+\exp \left(\beta_{\text {density }}+\beta_{\text {reciprocity }}\right)}
\end{gathered}
$$

where $\beta$ is a statistical parameter, of type indicated in the subscript. Equations (2) and (3) both include an initial fraction determining the probability of selecting a node, as well as a parameter for the density effect. However, (3) also includes a term for reciprocity because the probability of the tie from node $i$, the gray node in Figure A.1, that would form triad $021 \mathrm{C}(\mathrm{b})$ would be weighed against the option of forming a reciprocated tie, resulting in triad 102 (Figure A.1). Equation (2) does not contain this term because the tie from node $h$ that establishes triad $021 \mathrm{C}$ (a) does not have the potential to be a reciprocated tie. 
Thus, the logic of SAOMs allows us to mathematically represent each aspect of each node's local structure. In other words, SAOMs' "actor-oriented" approach captures nodes' embeddedness more fully than (L)ERGMs: network change in SAOMs depends upon both the macro-topology and the proximate context of each node. ${ }^{19}$

${ }^{19}$ See Block et al. 2016 for an extensive discussion of how SAOMs introduce greater dependency than ERGMs, as well as how SAOMs enable dependencies across network modes. 


\section{Appendix B Interview Data}

Christia (2012: 247-249) provides details of her interview procedures. Here, we briefly review some key aspects. Information on Balkh's militants was gathered by first compiling a list of militants operating in the region using administrative and secondary sources. Then, in the years following 2001, interviews were conducted with participants in the conflict-including many of the militants themselves, such as Balkh's prominent commander, Atta Muhammad Nur - and leading area experts. Interviews provided information on each militants' personal characteristics, influence, power, and actions. They were also used to crossvalidate responses from other interviews. Christia then identified militants' characteristics and behavior, such which militants formed alliances, during each of the well-defined and widely understood stages of the conflict. We use this information during specific periods to encode social ties by stage.

As mentioned in the main text, inferring social relationships from individuals' recollection can produce a biased measurement of social interactions (Bernard et al. 1979/1980; Killworth and Bernard 1979/1980). Our concerns are mitigated, however, by research indicating that recall can accurately and robustly capture typical interpersonal relational patterns (Romney and Faust 1982; Freeman and Romney 1987; Freeman

et al. 1987) and strong binary ties, such as alliances and divisions (Freeman et al. 1988; Ferligoj and Hlebec 1999; Hlebec and Ferligoj 2001). 


\section{Appendix C Topics and the Discursive Level}

Karell and Freedman (2019) explain the structural topic model (STM) used to identify 10 topics in the militants' writings. They also discuss the interpretation of topics at length. Since the primary aim of our article is to conceptualize and examine socio-semantic mechanisms rather than semantic content (see Martin 2002; Edelmann and Mohr 2018), we review the topics here rather than in the main text. Readers interested in the details of the topic model and further information on topical content should refer to Karell and Freedman 2019.

Figure C.2 presents the topic label and the 10 words within each topic with the highest FREX scores. ${ }^{20}$ These topics are estimated over the entire corpus. In brief, the topics indicate the following:

- Topic 1, "War", captures the idea of battling external enemies, such as enemy soldiers.

- Topic 5, "Afghan jihad (battle)", is similar to Topic 1. It also reflects the notion of external enemies like the Soviets and Najibullah Ahmadzaib. "Najib" was the leader of the communist regime in Kabul. In general, topics 1 and 5 refer to a meaning of jihad as an ongoing, externally-directed armed fight and political conflict (Nielsen 2017).

- Topic 7, "International politics", interprets the international arena as a site of contestation.

- Topic 8, "Social and political revolution", similarly to Topic 7, regards broad socio-political arenas as a site of "revolution".

- Topic 3, "Theology of jihad (struggle)", has much in common with topics 1, 5, 7, and 8 . It conveys the religious dimension of jihad-jihad as an ongoing struggle for godliness through spiritual purification (Nielsen 2017).

- Topic 4, "Local education and markets", reflects combatants' interest in everyday local, familial, and private matters, such as how one should treat various illnesses and educational policies and curricula. The "thousand" in this topic refers to prices - "ten thousand afghanis" - being set for different markets.

- Topic 6, "Religious code", comprises religiously-justified regulations of everyday life. Many documents associated with this topic are fatwas, or advisory legal opinions commonly used throughout Muslim societies.

- The remaining topics-Topics 2, 9, and 10 - are not clearly interpretable. We conceive of these as "residual categories", or collections of words that individually have meaning to combatants, but are grouped together due to their lack of fit in the other topics. For a discussion about residual categories and why topic model analyses often use a sub-set of topics, see Nielsen 2017.

${ }^{20}$ FREX scores are a measure of words' probability of appearing under a topic and their exclusivity to that topic (Roberts et al. 2014). 
Figure C.2: Topic Labels and Top FREX Words

1. War mujahideen, kill, attack, soldier, captur, enemi, oper, command, tank, area

2. Topic 2

3. Theology of jihad (struggle)

4. Local education \& markets educ, thousand, provinc, news, train, respect, ministry, school, abdul, mullah

5. Afghan jihad (battle) mujahedeen, jhad, soviet, najib, kabul, regim, afghan, elect, presid, pakistan

6. Religious code

7. International politics afghanistan, russian, countri, govern, unit, intern, russia, support, state, american

8. Social \& political revolution

9. Topic 9

10. Topic 10 god, jihad, quot, messeng, allah, brother, prophet, sheikh, bless, martyr

movement, holy, life, great, human, scholar, women, quran, answer, world social, uniti, polit, communiti, revolut, parti, west, exist, natur, system grant, sunday, catalog, prison, friday, inevit, servic, pose, pro, innov

pepe, pace, hair, crush, bottom, fashion, mesa, dam, code, matt

profil, breath, select, patent, post, bbc, threat, bar, tuesday, cost

Notes: Words have been stemmed. Figure taken from Karell and Freedman 2019. 


\section{Appendix D Summary of Researcher Decisions}

As with many other computational- and network-based methods for studying social and semantic space (e.g., Carley 1997; DiMaggio 2015; Kim and Diesner 2015; Nerghes et al. 2015; Nelson 2017), the analytical approach introduced in this article requires researchers to make several subjective decisions. In this appendix we highlight five important choices, mention tools and considerations that can help guide these choices, and point interested readers to further resources that discuss the decisions in more detail (Table D.1). Our list is not exhaustive, and our overall advice is for researchers to be transparent about their choices.

First, textual material must be preprocessed for use in topic models. Preprocessing decisions include, for example, whether to remove certain frequent words (some are commonly known as "stop words"), as well as special characters and numbers. A key choice involves how to parse the corpus into units of analysis, or "documents", such as paragraphs or pages. In our own analysis, our documents are pages due to decisions made by Karell and Freedman (2019). ${ }^{21}$ The specific choices that make up preprocessing are well known, and the consequences of these choices can be evaluated (e.g., Grimmer and Stewart 2013; Lucas et al. 2015; Denny and Spirling 2018).

The second decision regards the specification of topic models. For example, the researcher must select the number of topics to be estimated, commonly labeled $k$. In addition, if the researcher wants to include data on non-textual attributes of the document because these attributes might influence the topics, she can define covariates in a structural topic model (STM) (Roberts et al. 2014). In our study, we are interested in the concepts that constituted the semantic space of an episode lasting twenty years, so we use the results from an STM that included a temporal covariate (Karell and Freedman 2019).

While some view the flexibility of topic models, such as the option to select $k$, as potentially problematic, we see it is an opportunity to consider observable implications of the theory as part of validation. For socio-semantic network scholars, it also affords an opportunity to both develop and evaluate a theory that includes assumptions about non-textual influences on semantic space. As a result, we recommend that the specification of topic models be informed by the research question and, possibly, a theory (Nelson 2017), although researchers can alternatively - or in conjunction - use a data-driven approach to select $k$, as done in Light and Odden 2017.

The final three choices regard the definition of ties in the socio-semantic network. Researchers must decide how to code the semantic level ties among topics, as well as the cross-level ties between actors and topics. In addition, researchers need to identify cross-level ties as directed or undirected. In most cases, the researcher's theory will provide guidance. Here, we only provide comments and rules-of-thumb for determining inter-topic edges, cross-level ties, and the direction of cross-level ties.

\footnotetext{
${ }^{21}$ Karell and Freedman (2019) used pages as documents because it was the only consistent break-point across the kinds of publications in their corpus. All publications had pages breaks, whereas some publications had, say, paragraph breaks while others did not. Karell and Freedman evaluated the impact of this preprocessing decision by showing, first, that the main results remained largely unchanged when using documents based on breaks every 500 words, and, second, that the topics' semantic cohesion and exclusion decreased when using documents based on 150- and 350-word breaks.
} 
Table D.1: Researcher Decision-Points

\begin{tabular}{ll}
\hline Analytical step & Discussion \\
\hline $\begin{array}{l}\text { Preprocessing data for topic model } \\
\text { (including parsing documents) }\end{array}$ & $\begin{array}{l}\text { See Grimmer and Stewart 2013, Lucas et al. 2015, and } \\
\text { Denny and Spirling 2018; Example of documents: pages }\end{array}$ \\
$\begin{array}{l}\text { Specifying topic models (including } \\
\text { selecting number of topics, } k, \text { and } \\
\text { covariates for STMs) }\end{array}$ & $\begin{array}{l}\text { Theory-driven; See Roberts et al. 2014 and Nelson 2017; } \\
\text { for } k\end{array}$ \\
Coding ties between topics & $\begin{array}{l}\text { Theory-driven; Rule of thumb: test sensitivity to different } \\
\text { thresholds }\end{array}$ \\
Coding ties between actors and topics & $\begin{array}{l}\text { Theory-driven; Rule of thumb: prevalence of given topic in } \\
\text { author's documents should be above threshold equaling an } \\
\text { even prevalence of total topics } k \text { in documents }\end{array}$ \\
& $\begin{array}{l}\text { Theory-driven; Rule of thumb: actors make ties to topics } \\
\text { (i.e., directed from actors to topics) }\end{array}$ \\
\hline
\end{tabular}

In our analytic approach, ties between topics are based on their correlation within documents, across all documents of the corpus. The researcher's theory will likely help determine which correlation values should be coded as ties. For example, theories about weak and strong ties have clear implications for thresholds, and research questions about concepts being unlikely to be discussed together could employ negative correlation values. Light and Odden (2017: 14) select a threshold of 0.01, as we do, to uncover a backbone image of the network. In the absence of any theoretical expectations, we recommend that researchers conduct their analysis using the continuous correlation value. If binary values are needed, we suggest that researchers use different thresholds and compare their findings in a transparent way. This iteration through thresholds has the additional benefit of providing information on the sensitivity of results.

We advise researchers to exhibit care when interpreting edges among topics. A edge, or correlation between two topics, means that two clusters of words have some tendency to appear together in the documents of a corpus. In other words, because topics themselves already represent a tendency for words to appear together in documents, "network" associations between two topics are correlations between two frequency distributions of words, conditional on how words appear in documents. This is roughly akin to measuring "communities" using a network of words and then measuring the correlation across communities. However, in the "topical structure" approach, topic models do not make assumptions about structure at the wordlevel. In addition, at the topic-level, correlations are constrained by the documents. For instance, if a topic regularly comprises the entirety of words in the documents it appears in, it will not correlate with any other topic.

Thus, the inter-topic network provides two advantages for representing (textual) semantic space. First, the researcher does not need to make strong assumptions about network structure among words. Instead, 
groupings of word usage are identified, and words can appear in multiple groups. Second, if the documents are defined in a manner that reflect individuals' consumption of the text (e.g., pages of a newspaper can capture the consumption of "front page news"), then the ties between topics approximate how humans may be understanding intra-topic connections.

Researchers also face a decision when coding ties between actors and topics. These cross-level ties are based on the prevalence of topics in a specific actor's, or author's, collection of documents at a given point in time. The researcher must determine what threshold of prevalence counts as a tie. A reasonable threshold is a value greater than the topic-proportion value if each topic contributed equally to the composition of the entire corpus. For example, if there are 10 topics that in some combination compose the corpus, a tie can be said to exist between an author and a topic if the prevalence of a given topic in the author's documents is greater than 10 percent.

While theory will also likely guide the coding of cross-level ties' directionality, researchers can begin by coding ties as emanating from actors and being received by topics. This default direction is based on the assumption that people and organizations have the agency in forming links to semantic elements. Researchers can easily compare results with this specification to results from analyses using undirected or reciprocated cross-level ties (e.g., Snijders et al. 2013; Hollway et al. 2017).

Finally, we highlight an obvious but very important consideration that occurs before implementing our approach. Namely, the definition of the population of semantic material from which researchers sample textual data predetermines the findings (Lee and Martin 2015). In our reading of the computational textual analysis literature, this consideration appears to not be as deeply explored the as it usually is in comparative-historical sociology, cultural sociology, and archival studies. (With regards to our own study, see the discussion in Karell and Freedman 2019). 


\section{Appendix E Graphical Representation of Network Data}

Figure E.3: Socio-Semantic Graph Across Time

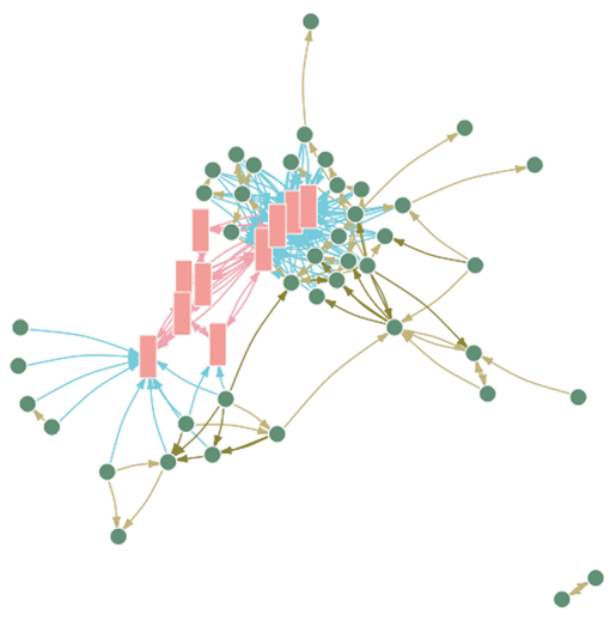

(a) Wave 1: Early Jihad

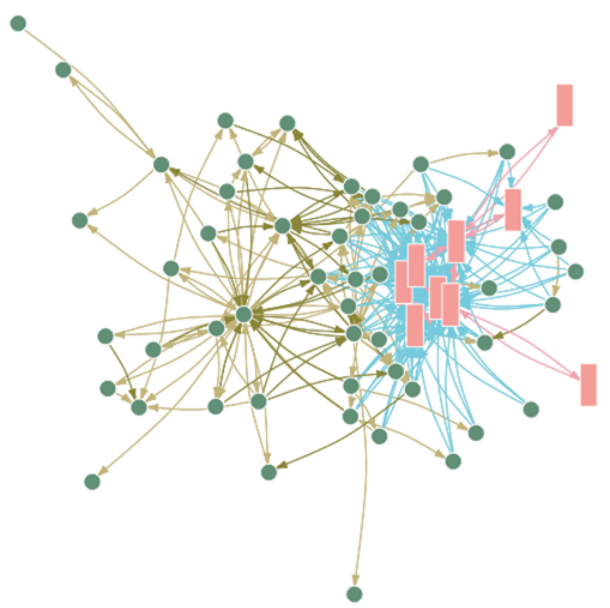

(c) Wave 3: Civil war

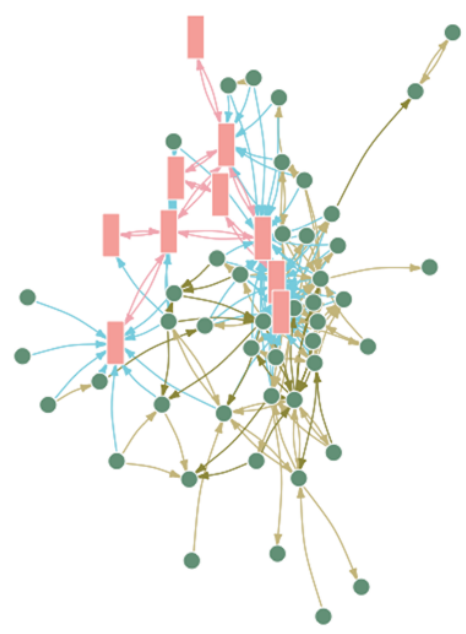

(b) Wave 2: Late Jihad

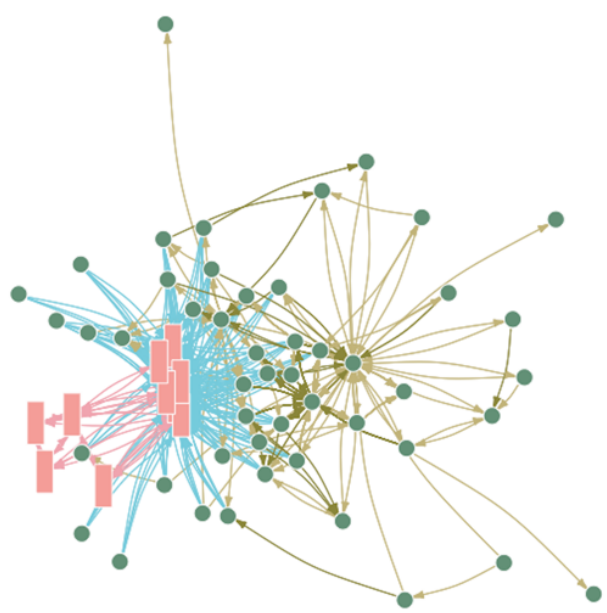

(d) Wave 4: Taliban era

Notes: Circular vertices represent militants and rectangles represent topics. Light brown and dark brown edges indicate comrade and enemy relationships between militants, respectively. Blue edges denote ties between militants and topics. Pink edges are ties between topics. Node layout is not constant across waves. 


\section{Appendix F Goodness of Fit}

We assess the models' goodness of fit using the procedures outlined in Leifeld and Cranmer 2016 (see also Lospinoso 2012). For each wave, estimated parameters are used to simulate the transition of networks from the preceding period. Then, we compare unmodeled endogenous features of the simulated networks to the same features in the observed network. ${ }^{22}$ Following the current convention of the stochastic actor-oriented model (SAOM) scholarship (e.g., Block et al. 2016), we evaluate our social level networks with three unmodeled statistics: indegree, outdegree, and a triadic census. ${ }^{23}$

Studies using SAOMs to analyze two-mode and undirected graphs are relatively less common. We assess the cross-level fit using the mixed triadic census proposed by Hollway et al. (2017), which compares the simulated and observed triads formed by the ties linking nodes across levels. Finally, we evaluate the nondirected semantic network with four statistics: degree, k-stars, cycles, and a triad census.

We observe good fit overall. The assessments for Model 3, our complete model, are presented in Figure F.4, Figure F.5, and Figure F.6. The plots in Figure F.4 and Figure F.6 each compare the frequency distribution of one network statistic of the simulated networks, indicated by the gray boxplots, to the mean observed frequency of the same statistic, represented by the black line, across values of the statistic. The horizontal axis of each plot indicates the value of the statistic; the vertical axis shows the frequency of the simulated and observed statistic at a given value. Figure F.5 is similar. The simulated values of each kind of triad is shown by the box and violin plots, with the dashed gray line depicting the $95 \%$ confidence interval, and the solid line and figures are the observed values. ${ }^{24}$

For each of our assessments, the more a model has similar simulated and observed frequencies across values of each statistic, the better the fit. All of the goodness of fit plots are accompanied by statistical tests of difference between the simulated and observed network features across values of the distribution. The results are these tests are available from the authors upon request.

Figure F.4 shows the excellent fit achieved for the comrade and enemy networks. The observed frequencies fall within the range of simulated frequencies for all values of each statistic. The mixed triad census (Figure F.5) indicates that the model fits the cross-level network less well. We do achieve good fit for several elementary configurations that underpin multilevel dependencies (Hollway et al. 2017): a tie between the focal actor and cultural element, with reciprocated ties between the focal actor and an alter (triad "12"); a tie between the focal actor and cultural element, with an non-reciprocated tie outgoing from the focal actor (triad "11D"); a tie between the focal actor and discursive element, with an non-reciprocated tie incoming to the focal actor (triad "11U"); and reciprocation between actors not sharing a cultural affiliation (triad "02"). We also achieve satisfactory fit for the higher order triad of reciprocation between actors sharing a cultural affiliation (triad "22"). We are unsure why the remaining triad kinds are not modeled well; different

\footnotetext{
${ }^{22}$ The procedure is implemented using xergm version 1.8.2 (Leifeld et al. 2017) with R 3.3.4.

${ }^{23}$ We do not assess goodness of fit using an out-of-sample prediction of tie location, as proposed by Leifeld and Cranmer (2016), because of the theoretical concerns raised by Block et al. (2018).

${ }^{24}$ For a detailed explanation of the plot format, see Lospinoso 2012 and Hollway et al. 2017.
} 
specifications of the model with a range of cross-level effects did not lead to improvements. However, because several triads in the cross-level network are modeled well and the fit of the other three networks is very good, we remain confident in our findings. Indeed, Figure F.6 shows that our model fits the topic network very well. For each network statistic, there are no statistical differences between the simulated and observed frequencies for the majority of values. 
Figure F.4: Goodness of Fit for the Comrade and Enemy Networks
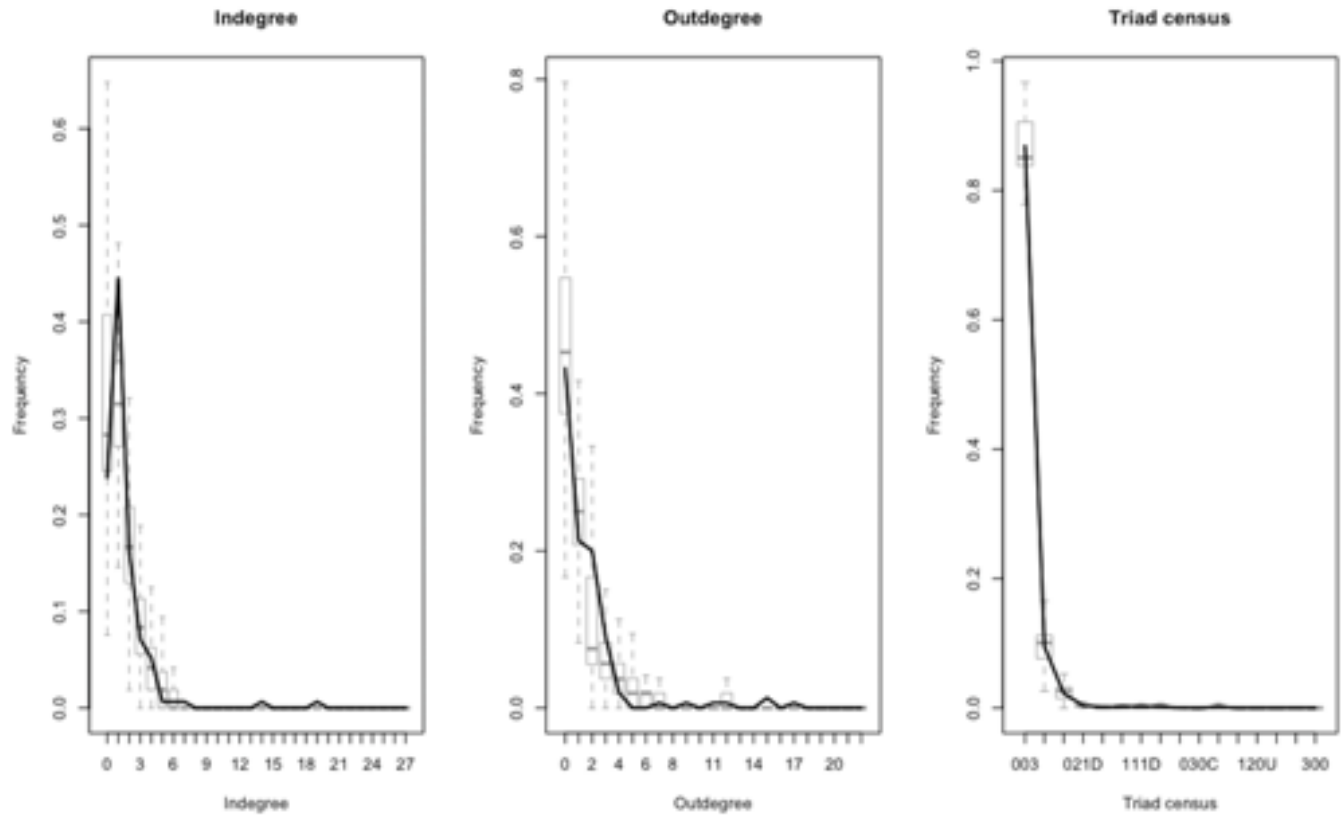

(a) Comrade network statistics
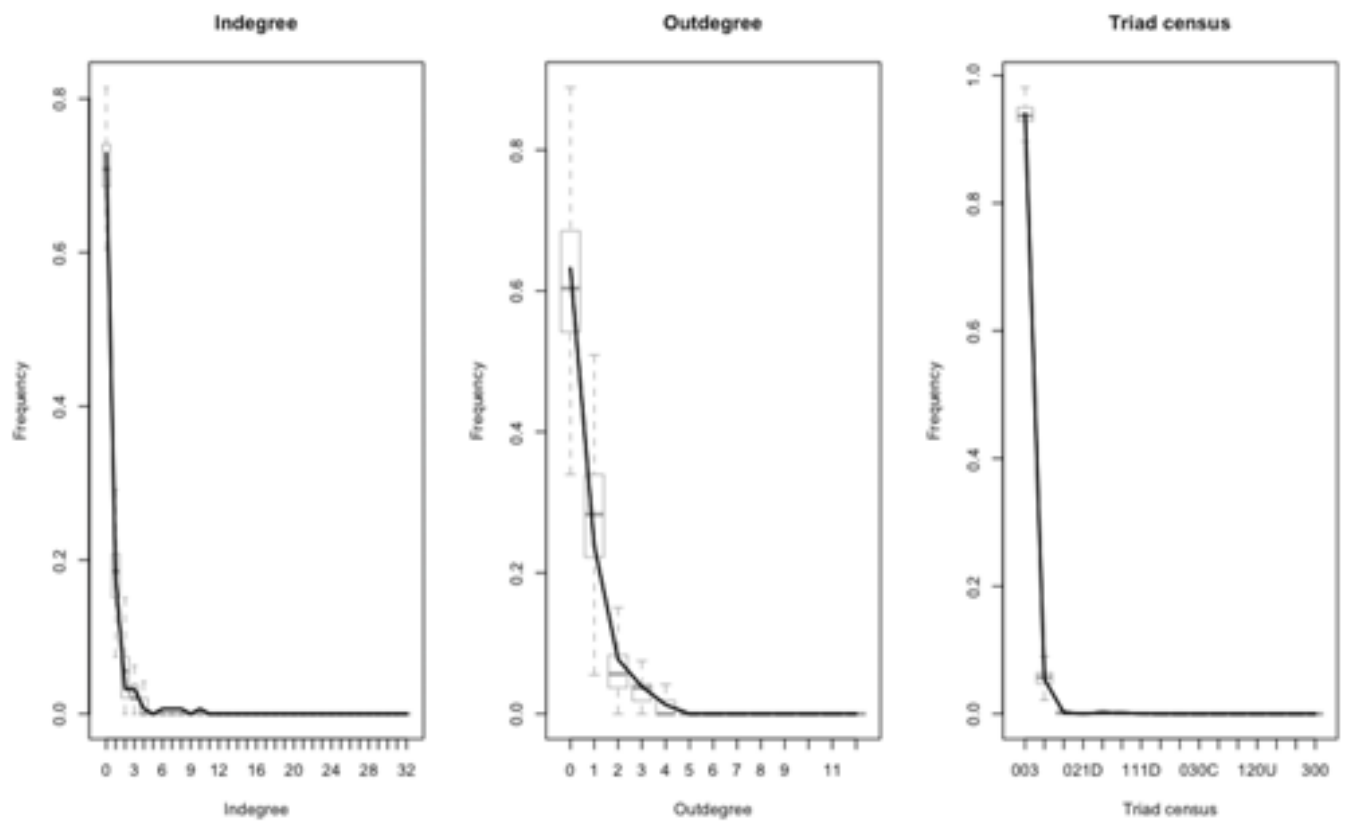

(b) Enemy network statistics

Notes: Box plots show the frequency distribution of the simulated statistic for each value of the statistic. The solid line depicts the mean observed frequency of the statistics across values. 
Figure F.5: Multilevel Model Mixed Triad Census Goodness of Fit

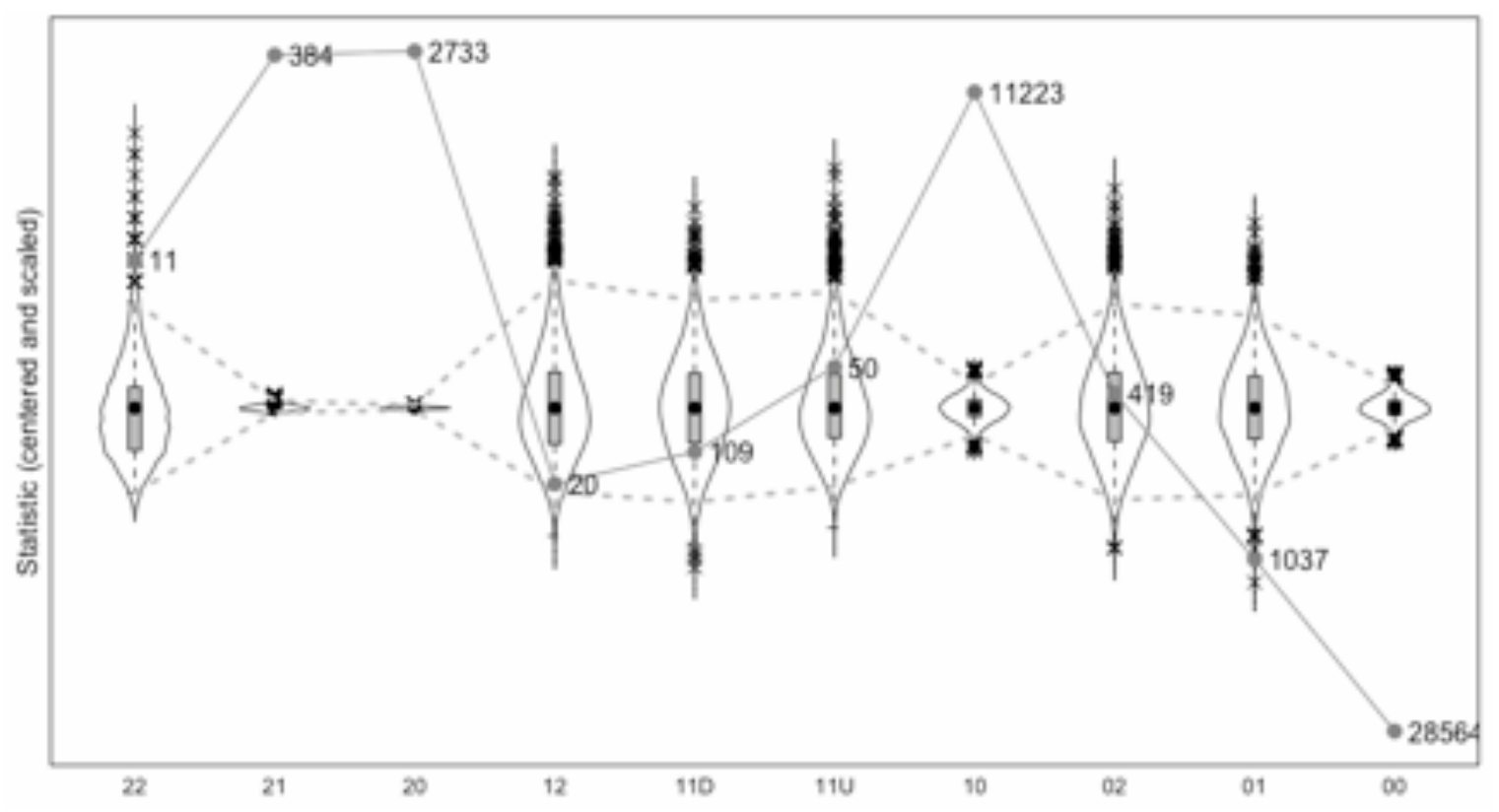

Notes: The first digit of the triad label indicates the number of edges between levels. The second digit tells the number and direction of edges in the social level. The box and violin plots show the frequency distribution of each kind of triad in the simulated networks and the solid line indicates the observed frequency. 
Figure F.6: Goodness of Fit for the Discursive Network
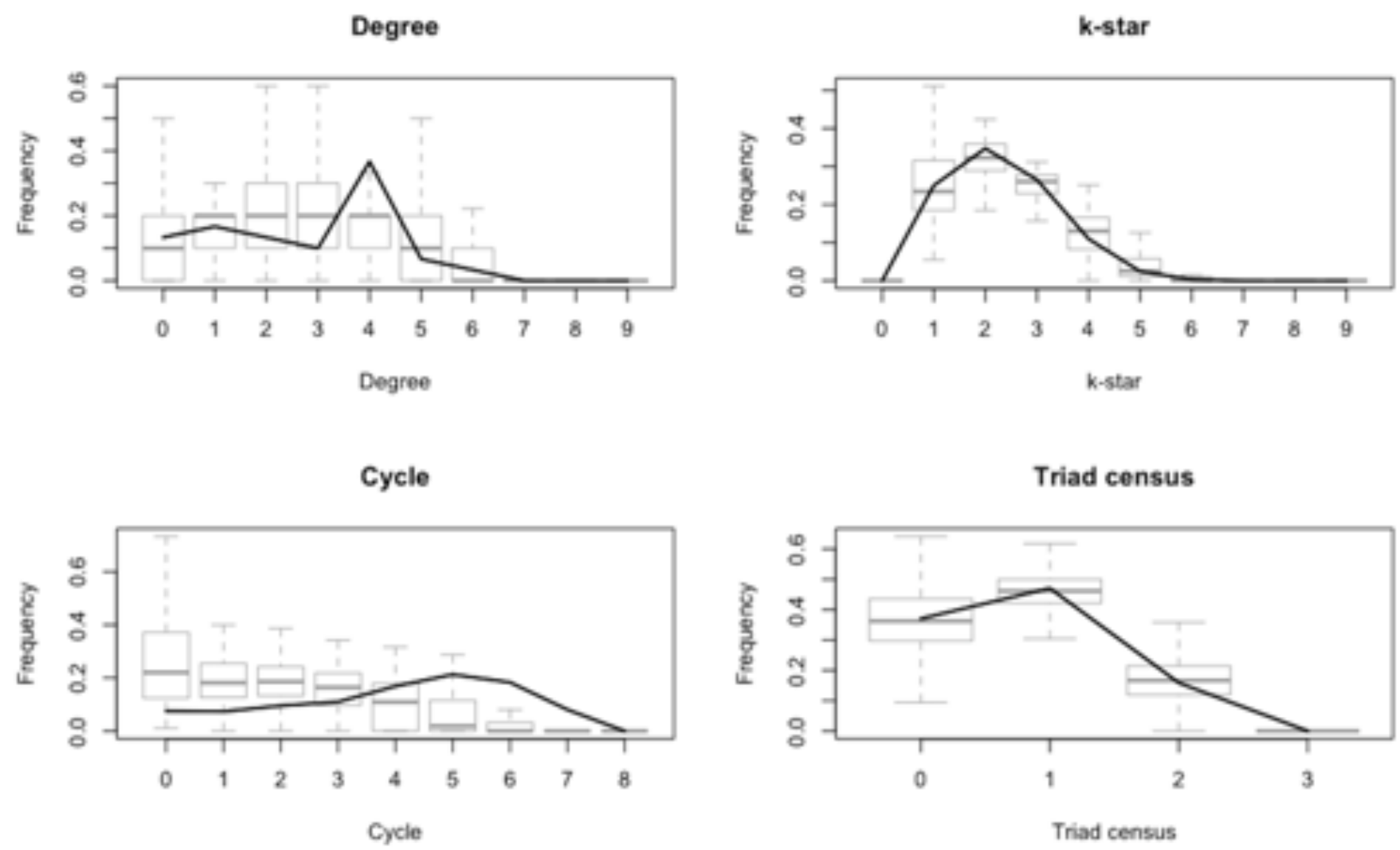

Notes: Box plots show the frequency distribution of the simulated statistic for each value of the statistic. The solid line depicts the mean observed frequency of the statistics across values. 